\section{Abstract}

\title{
Saturn Ring Rain: Model Estimates of Water Influx into Saturn's Atmosphere
}

\author{
L. Moore ${ }^{1}$, J. O’Donoghue ${ }^{2}$, I. Mueller-Wodarg ${ }^{3}$, M. Galand ${ }^{3}$, and M. Mendillo ${ }^{1,4}$ \\ ${ }^{1}$ Center for Space Physics, Boston University, Boston, Massachusetts, USA \\ ${ }^{2}$ Department of Physics and Astronomy, University of Leicester, Leicester, UK \\ ${ }^{3}$ Space and Atmospheric Physics Group, Imperial College London, London, UK \\ ${ }^{4}$ Department of Astronomy, Boston University, Boston, Massachusetts, USA
}

Recently $\mathrm{H}_{3}{ }^{+}$was detected at Saturn's low- and mid-latitudes for the first time (O'Donoghue et al., 2013), revealing significant latitudinal structure in $\mathrm{H}_{3}{ }^{+}$emissions, with local extrema in one hemisphere mirrored at magnetically conjugate latitudes in the opposite hemisphere. The observed minima and maxima were shown to map to regions of increased or decreased density in Saturn's rings, implying a direct ring-atmosphere connection. Here, using the Saturn Thermosphere Ionosphere Model (STIM), we investigate the "ring rain" explanation of the O'Donoghue et al. (2013) observations, wherein charged water group particles from the rings are guided by magnetic field lines as they "rain" down upon the atmosphere, altering local ionospheric chemistry. Based on model reproductions of observed $\mathrm{H}_{3}{ }^{+}$variations, we derive maximum water influxes of (1.6-16) $\times 10^{5} \mathrm{H}_{2} \mathrm{O}$ molecules $\mathrm{cm}^{-2} \mathrm{sec}^{-1}$ across ring rain latitudes $\left(\sim 23-49^{\circ}\right.$ in the south, and $\sim 32-54^{\circ}$ in the north), with localized regions of enhanced influx near $48^{\circ},-38^{\circ}, 42^{\circ}$, and $53^{\circ}$ latitude. We estimate the globally averaged maximum ring-derived water influx to be (1.6-12)x $10^{5} \mathrm{~cm}^{-2} \mathrm{sec}^{-1}$, which represents a maximum total global influx of water from Saturn's rings to its atmosphere of (1.0-6.8) $\times 10^{26} \mathrm{sec}^{-1}$. The wide range of global water influx estimates stems primarily from uncertainties regarding $\mathrm{H}_{3}{ }^{+}$temperatures (and consequently column densities). Future ring rain observations may therefore be able to reduce these uncertainties by determining $\mathrm{H}_{3}{ }^{+}$temperatures self consistently. 


\section{Introduction}

\subsection{Water in Saturn's Ionosphere}

A source of exogenous water has long been inferred at Saturn, particularly as a means to

31 reduce calculated ionospheric densities in order to reproduce observed values. Early Saturn

32 ionospheric models (e.g., McElroy, 1973; Capone et al., 1977) predicted electron densities an

33 order of magnitude larger than those later measured by the Pioneer 11 and Voyager spacecraft

34 (Kliore et al., 1980; Kaiser et al., 1984; Lindal et al., 1985). One chemical effect of introducing

35 oxygen bearing compounds into Saturn's upper atmosphere is to convert $\mathrm{H}^{+}-$a long-lived major

36 atomic ion in outer planet ionospheres - into a short-lived molecular ion that quickly

37 dissociatively recombines, thereby reducing the net electron density.

38 While a number of modeling studies have been able to derive a range of water influxes

39 that adequately explain the Pioneer and Voyager radio occultation measurements (e.g.,

40 Connerney and Waite, 1984; Majeed and McConnell, 1991, 1996), directly constraining the

41 influxes observationally has proven more difficult. The first unambiguous direct detection of

42 water in Saturn's upper atmosphere came from the Infrared Space Observatory (ISO;

43 Feuchtgruber et al., 1997), which measured an $\mathrm{H}_{2} \mathrm{O}$ column abundance of $(0.8-1.7) \times 10^{15} \mathrm{~cm}^{-2}$

44 and was used to derive a global water influx of $\sim 1.5 \times 10^{6} \mathrm{H}_{2} \mathrm{O}$ molecules $\mathrm{cm}^{-2} \mathrm{sec}^{-1}$ (Moses et al.,

45 2000). Subsequent studies based on Submillimeter Wave Astronomy Satellite and Herschel

46 Space Observatory measurements found global influx values within a factor of 2 of the Moses et

47 al. results (Bergin et al., 2000; Hartogh et al., 2011). Despite predictions of latitudinally varying

48 water influxes (e.g., Connerney, 1986), no observational confirmation of such variations has

49 been made to date, with only ambiguous detections of latitudinally varying water concentrations

50 in the ultraviolet (e.g., a $2 \sigma$-detection of $2.70 \times 10^{16} \mathrm{~cm}^{-2}$ at $33^{\circ} \mathrm{S}$ latitude: Prangé et al., 2006), 
51 and preliminary indications of larger equatorial water densities from Cassini Composite InfraRed

52 Spectrometer (CIRS) observations (Bjoraker et al., 2010).

53 A counter-intuitive trend in electron density with latitude was revealed after the arrival of

54 Cassini at Saturn, based on the 31 new radio occultation measurements published to date (Nagy

55 et al., 2006; Kliore et al., 2009). Despite being near Saturn's equinox, with the sun directly

56 overhead at low-latitudes, electron densities were found to be lowest at Saturn's equator and to

57 increase with latitude, a behavior that Moore et al. (2010) were able to reproduce by introducing

58 a water influx that peaked at Saturn's equator and decreased with latitude. Such a water influx

59 profile is in agreement with predictions by models investigating the evolution of Enceladus'

60 water vapor plumes (Jurac and Richardson, 2007; Cassidy and Johnson, 2010; Fleshman et al.,

61 2012). While a ring-derived ionized influx may yet be present, there are only five published

62 Cassini radio occultations at latitudes that map magnetically to Saturn's rings, and therefore there

63 is insufficient latitudinal resolution in the electron density observations to clearly identify any of

64 the expected local extrema that would result from possible ring-derived influxes.

66 1.2. Saturn Ring Rain Observations

67 Recently $\mathrm{H}_{3}{ }^{+}$was detected at Saturn's low- and mid-latitudes for the first time 68 (O'Donoghue et al., 2013), revealing significant latitudinal structure in $\mathrm{H}_{3}{ }^{+}$emissions, with local

69 extrema in one hemisphere mirrored at magnetically conjugate latitudes in the opposite

70 hemisphere. Furthermore, the observed minima and maxima were shown to map to regions of

71 increased or decreased density in Saturn's rings, implying a direct ring-atmosphere connection.

72 The $\mathrm{H}_{3}{ }^{+}$ion has a relatively short chemical lifetime; its dominant loss process is dissociative

73 recombination with electrons. While oxygen bearing compounds such as $\mathrm{OH}$ and $\mathrm{H}_{2} \mathrm{O}$ have 
74 typically been introduced into models of Saturn's ionosphere as a means of reducing the electron

75 density through charge exchange with $\mathrm{H}^{+}$, they also impact $\mathrm{H}_{3}{ }^{+}$densities, primarily by reducing

76 dissociative recombination rates. Therefore, the sharp latitudinal structures observed by

77 O'Donoghue et al. (2013) - which cannot be explained by solar ionization effects - likely

78 represent a proxy for external oxygen influxes (Connerney, 2013). Here, using the Saturn

79 Thermosphere Ionosphere Model (STIM), we estimate the upper limits for ring-derived

80 atmospheric ion influxes implied by the ring rain observations, and we use those values to derive

81 lower limits on ring mass loss rates.

82

\section{2. Methods}

\section{2.1. Ring Ion Influx}

85 Saturn's rings are composed primarily of water ice bodies (e.g., Cuzzi et al., 2010 and

86 references therein) between $1 \mathrm{~cm}$ and $20 \mathrm{~m}$ in size (Zebker et al., 1985; French and Nicholson,

87 2000). Solar UV photon-induced decomposition of ice leads to the production of an $\mathrm{O}_{2}$ ring

88 atmosphere, which can accumulate both above and below the ring plane due to a long lifetime

89 and frequent interactions with ring particles (Johnson et al., 2006). Two Cassini instruments

90 detected a ring ionosphere during Cassini's orbital insertion in 2004, finding evidence of $\mathrm{O}^{+}$and

$91 \mathrm{O}_{2}{ }^{+}$ions (Tokar et al., 2005; Waite et al., 2005), likely the result of photoionization of $\mathrm{O}_{2}$.

92 Models of Saturn's ring ionosphere support the dominance of $\mathrm{O}^{+}$and $\mathrm{O}_{2}{ }^{+}$, and further find that

93 within the radius where Keplerian and corotation velocities are equal in the ring plane, $\sim 1.8 \mathrm{R}_{\mathrm{S}}$,

94 ring ions spiral along magnetic field lines and precipitate into Saturn's atmosphere with near unit

95 efficiency. Outside of this radius initially trapped ions can also later be scattered into the loss

96 cone (Luhmann et al., 2006; Tseng et al., 2010). Therefore, as $\mathrm{O}_{2}{ }^{+}$and $\mathrm{O}^{+}$are the dominant ring 
97 ionosphere ions, they are also the ring-derived ions most likely to precipitate into Saturn's 98 atmosphere.

$99 \quad \mathrm{As}_{2}{ }^{+}$ions dissociatively recombine with electrons extremely rapidly - roughly three

100 times faster than $\mathrm{H}_{3}{ }^{+}$ions in Saturn's ionosphere - any precipitating $\mathrm{O}_{2}{ }^{+}$will lead to a chain of 101 photochemical reactions that produce primarily $\mathrm{OH}$ (via $\mathrm{O}+\mathrm{H}_{2}$ ) and $\mathrm{H}_{2} \mathrm{O}$ (via $\mathrm{OH}+\mathrm{H}_{2}$ ) in the

102 thermosphere and lower atmosphere (e.g. Moses et al., 2000). An influx of $\mathrm{H}_{2} \mathrm{O}$ or $\mathrm{H}_{2} \mathrm{O}^{+}$would 103 lead to similar chemistry, as the products of dissociative recombination reactions between $\mathrm{H}_{3} \mathrm{O}^{+}$

104 (formed rapidly from $\mathrm{H}_{2} \mathrm{O}^{+}$) and electrons include $\mathrm{OH}$ and $\mathrm{H}_{2} \mathrm{O}$. In other words, an external flux 105 of neutrals (e.g., $\mathrm{H}_{2} \mathrm{O}$ ) or ions (e.g., $\mathrm{O}_{2}^{+}$) can lead to similar number densities of oxygen-bearing 106 molecules in Saturn's atmosphere, and it is these molecules that charge-exchange with $\mathrm{H}^{+}$. The 107 subsequent rapid dissociative recombination of the resulting charge-exchange products (e.g., $108 \mathrm{H}_{3} \mathrm{O}^{+}$) then leads to the required reduction in Saturn's electron densities and the subsequent 109 decrease in $\mathrm{H}_{3}{ }^{+}$chemical loss rates. The most important remaining distinction between ionized 110 and neutral influxes is their deposition latitude, as ions are constrained to precipitate along 111 magnetic field lines. Therefore, in order to maintain consistency with previous Saturn 112 ionospheric literature, we treat the ring rain influx inferred from $\mathrm{H}_{3}{ }^{+}$observations here as a 113 "water" influx. In this way derived fluxes can be compared directly with previous results, and 114 the language of this text is simplified. Finally, it should be noted that water from Saturn's rings 115 may also be more efficiently transported in the form of charged sub-micrometer grains rather 116 than ions (e.g., Connerney, 2013), a possibility that remains to be evaluated.

\subsection{Temperatures in Saturn's Upper Atmosphere}

119 The observations of O'Donoghue et al. (2013) report the intensity of $\mathrm{H}_{3}{ }^{+}$emission (in $120 \mathrm{nW} \mathrm{m} \mathrm{m}^{-2}$ ) versus planetocentric latitude. (All latitudes quoted in this manuscript are 
121 planetocentric unless specified otherwise.) In order to compare those observations with model 122 results, a conversion between intensity and vertical column content is required. Typically, the 123 intensity of two or more discrete ro-vibrational spectral lines of $\mathrm{H}_{3}{ }^{+}$can be used to determine its 124 temperature and subsequently its density (Miller et al., 2000). The O'Donoghue et al. 125 observations unfortunately lacked sufficient signal-to-noise to carry out such a derivation. 126 Therefore, in order to estimate the $\mathrm{H}_{3}{ }^{+}$temperatures that correspond with the ring rain 127 observations, we follow a three step process: (1) determine the most realistic representation of 128 the behavior of neutral exospheric temperature with planetocentric latitude, $T_{\text {exo }}\left(\phi_{p c}\right)$, based on 129 ultraviolet solar and stellar occultations; (2) use STIM to find the temperature differential 130 between the exobase and the altitude of $\mathrm{H}_{3}{ }^{+}$ions (typically $\sim 2700-3000 \mathrm{~km}$ and $\sim 1200 \mathrm{~km}$ above 131 the 1 bar pressure level at Saturn, respectively); and (3) apply the STIM temperature differential 132 to a functional form of $T_{\text {exo }}\left(\phi_{p c}\right)$ (see below), yielding neutral temperature predictions at $\mathrm{H}_{3}{ }^{+}$ 133 altitudes, $T_{H_{3}^{+}}\left(\phi_{p c}\right)$.

134 Early Voyager analyses of solar and stellar occultations suggested that Saturn's 135 exospheric temperature could be as high as $850 \mathrm{~K}$ (Broadfoot et al., 1981; Festou and Atreya, 136 1982). However, subsequent Voyager 2 reanalyses found a temperature closer to $420 \mathrm{~K}$ (Sandel 137 et al., 1982; Smith et al., 1983), a value also supported by modern reanalyses of occultations by 138 both Voyager spacecraft (Vervack and Moses, 2013). Given the limited number of Voyager 139 observations and the uncertainties in analyzing them, it was not possible to use them to construct 140 a complete latitudinal trend for Saturn upper atmospheric temperatures. However, recent 141 analyses of 15 solar (Koskinen et al., 2013) and 3 stellar (Shemansky and Liu, 2012) occultations 142 by Cassini, in combination with previous Voyager results, now allow for a more realistic 143 estimate of the behavior of upper atmosphere temperature with latitude. 
Figure 1 presents the upper atmosphere temperature measurements described above

145 (aside from the values above $800 \mathrm{~K}$ ), as well as some auroral $\mathrm{H}_{3}{ }^{+}$temperature measurements

146 (Melin et al., 2007, 2011; O’Donoghue et al., 2014), a neutral temperature proxy at lower

147 altitudes. Different symbols represent the references highlighted in the figure legend, gray

148 vertical lines indicate quoted or estimated temperature uncertainties, and the gray shaded regions

149 highlight the latitudes of ring rain observations (from Figure 2 of O'Donoghue et al., 2013).

150 Figure 1 also presents a number of different methods for deriving $T_{\text {exo }}\left(\phi_{p c}\right)$ from the

151 measurements. Orange and green lines result from least square fits to the function $T_{\text {exo }}\left(\phi_{p c}\right)=$

$152 A_{1} \sin ^{2} \phi_{p c}+A_{2} \cos ^{2} \phi_{p c}$, where $A_{l}$ and $A_{2}$ are constants that vary according to the

153 combinations of datasets that have been included in each fit (as indicated by the numbers in

154 brackets). These curves are labeled as $\mathrm{S} 0$ and $\mathrm{S} 1$, and will be referred to using those

155 designations for the remainder of the text. The dotted line is for a linear least squares fit (fit L1),

156 while the dashed line represents the global arithmetic mean $T_{\text {exo }}$ (i.e., assumed constant with

157 latitude; fit M1). Data points are weighted by the inverse square of their uncertainties in order to

158 derive the various fits. Finally, cyan and purple lines correspond to the maximum and minimum

159 temperatures from the above fits, respectively, within the ring rain latitude regions (fits $\mathrm{T}_{\mathrm{MAX}}$ and

$\left.160 \mathrm{~T}_{\mathrm{MIN}}\right)$.

161 Two main points should be emphasized from Figure 1. First, there is the drastic

162 difference in $T_{e x o}\left(\phi_{p c}\right)$ profiles between fits that do and do not include the Cassini solar 163 occultations values (Koskinen et al., 2013). These are labeled as reference [6] in the figure

164 legend, and their dominance in derivations of meridional temperature trends emphasizes their 165 value in understanding the energetics of Saturn's upper atmosphere. Second, despite the 166 additional insight brought by the Cassini occultations, there remains significant uncertainty 
167 and/or variability in global thermospheric temperature determinations. It is, perhaps, not

168 surprising that there is no obvious global trend in neutral temperature, as measurements span a

169 range of seasonal and solar conditions at Saturn. Different researchers may well favor different

170 methods for constructing a "true" global thermospheric temperature behavior from the limited

171 available data. Therefore, the cyan and purple lines, representing a maximum and minimum

172 temperature at ring rain latitudes, respectively, will be used to illustrate how different choices

173 regarding ring rain exospheric temperatures would affect the subsequent results, such as

174 estimated $\mathrm{H}_{3}{ }^{+}$column densities and ring-derived water influxes.

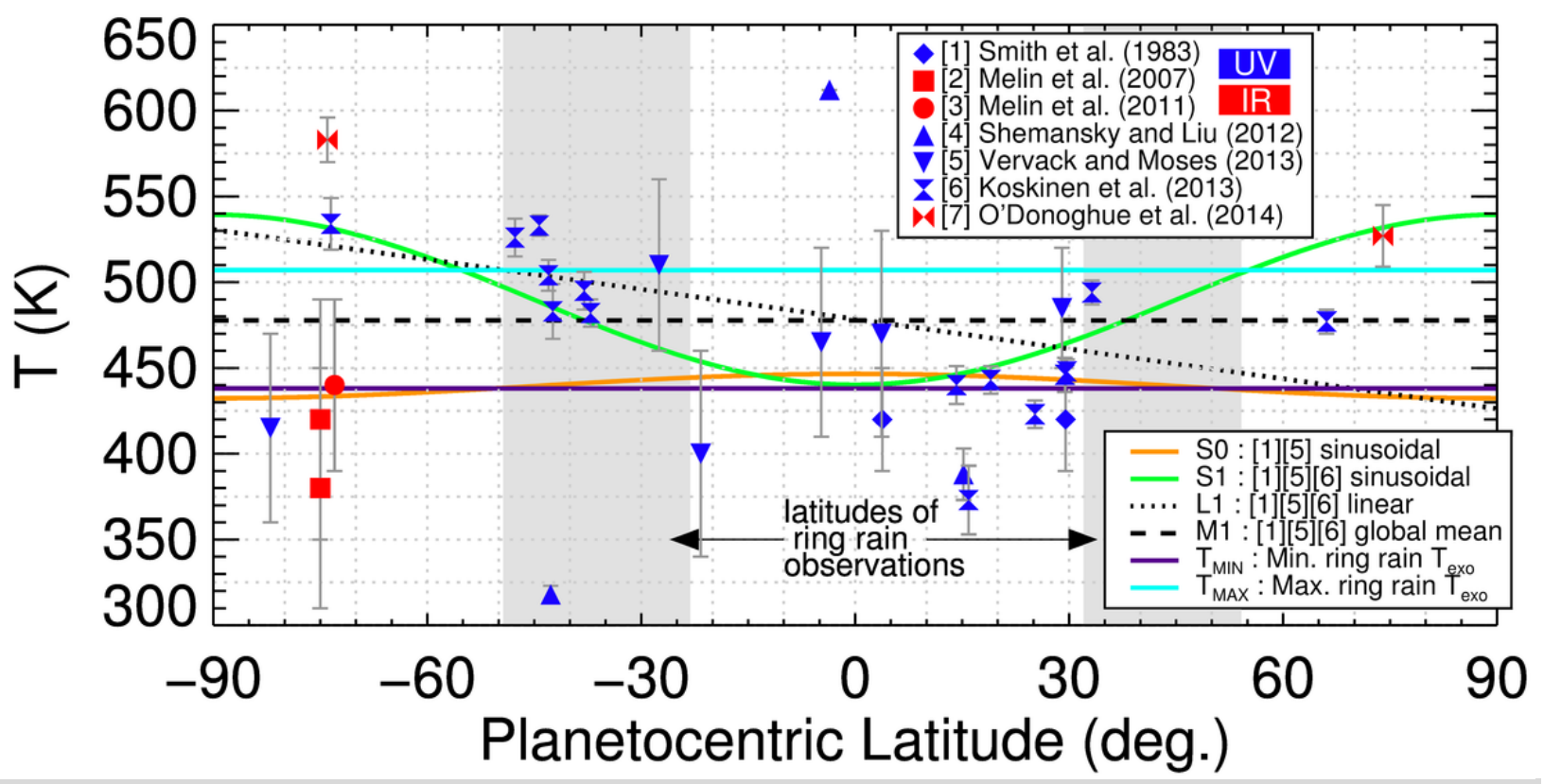

Figure 1. Symbols represent thermospheric temperature measurements from ultraviolet stellar and solar occultations - references [1], [4], [5], [6], blue symbols - and from auroral infrared $\mathrm{H}_{3}{ }^{+}$ observations - references [2], [3], and [7], red symbols. Curves indicate Global temperature fits that weight the data points by the inverse square of their uncertainties. Green and orange lines (fits S0 and S1) assume a functional form $A_{1} \sin ^{2} \phi_{p c}+A_{2} \cos ^{2} \phi_{p c}$, the dotted curve is for a linear fit (L1), and the dashed curve is the global mean temperature (M1). Cyan and purple lines correspond to the maximum and minimum temperatures from the fits at ring rain latitudes, indicated by gray shaded regions.

Some additional discussion regarding the choice of fits in Figure 1 is worthwhile. First,

$176 \mathrm{H}_{3}{ }^{+}$temperatures would ideally be taken directly from $\mathrm{H}_{3}{ }^{+}$observations. Unfortunately, previous

177 measurements of $\mathrm{H}_{3}{ }^{+}$temperatures are only available at auroral latitudes, and therefore don't 
178 provide adequate insight into temperatures at ring rain latitudes. Moreover, as $\mathrm{H}_{3}{ }^{+}$temperature 179 measurements sample a lower altitude region than the UV temperature measurements, additional 180 assumptions regarding IR-UV temperature differentials would be necessary to include them in 181 any global temperature fit. Instead, the available $\mathrm{H}_{3}{ }^{+}$temperature measurements are included in 182 Figure 1 for completeness, and for comparison with UV temperature measurements. Second, the 183 Cassini stellar occultation results of Shemansky and Liu (2012) have been omitted from the 184 temperature fits of Figure 1 due to the lack of published uncertainty for the $\beta$ Cru occultation at $1853.6^{\circ}$ latitude (blue triangles in Figure 1). If an uncertainty of $10 \mathrm{~K}$ were assumed for the $\beta \mathrm{Cru}$ 186 measurement - midway between the $5 \mathrm{~K}$ and $15 \mathrm{~K}$ uncertainties reported at $-42.7^{\circ}$ and $15.2^{\circ}$ 187 latitude (Shemansky and Liu, 2012) - then a sinusoidal fit using references [1], [4], [5] and [6] 188 would yield a temperature profile roughly constant with latitude, near $460 \mathrm{~K}$, and well within the 189 temperature range between the cyan and purple lines of Figure 1.

190 Observed ro-vibrational infrared emissions from the $\mathrm{H}_{3}{ }^{+}$ion are dependent on 191 temperature and density of the emitting gas. Neutral temperature in Saturn's thermosphere starts 192 at a lower boundary value of $\sim 150 \mathrm{~K}$ near the homopause (e.g., Hubbard et al., 1997), and 193 increases towards an isothermal value (the "exospheric temperature") in the upper thermosphere. $194 \mathrm{As} \mathrm{H}_{3}{ }^{+}$is expected to be in quasi-thermal equilibrium with the surrounding gas in the outer 195 planets at lower altitudes (Miller et al., 1990; Moore et al., 2008), its emissions are thus a good 196 proxy for neutral temperatures over the altitude regions where $\mathrm{H}_{3}{ }^{+}$densities peak. Ionospheric 197 models predict the altitude of maximum $\mathrm{H}_{3}{ }^{+}$density to be between $~ 1000-1400 \mathrm{~km}$, depending 198 on solar and seasonal conditions and latitude (Majeed and McConnell, 1996; Moses and Bass, 199 2000; Moore et al., 2009). Recently, the auroral $\mathrm{H}_{3}{ }^{+}$emission was observed to peak near 1155 $200 \mathrm{~km}$ (Stallard et al., 2012), essentially coincident with a previously measured UV auroral 
201 emission altitude (Gérard et al., 2009). Therefore, at least in auroral regions, measured $\mathrm{H}_{3}{ }^{+}$ 202 temperatures appear to primarily sample temperatures in the lower thermosphere, and are 203 expected to be up to $50 \mathrm{~K}$ cooler than the corresponding exospheric temperatures (Müller204 Wodarg et al., 2012).

205 In order to convert the $T_{\text {exo }}\left(\phi_{p c}\right)$ profiles derived in Figure 1 into $\mathrm{H}_{3}{ }^{+}$temperature 206 profiles we turn to the $T_{\text {exo }}\left(\phi_{p c}\right)-T_{H_{3}^{+}}\left(\phi_{p c}\right)$ temperature differentials calculated by the Saturn 207 Thermosphere Ionosphere Model (STIM; Müller-Wodarg et al., 2012). STIM is a General 208 Circulation Model (GCM) that treats the global response of Saturn's upper atmosphere to solar 209 and auroral forcing. Key physical quantities calculated in STIM include neutral temperatures, 210 neutral and ion densities, neutral winds, and ion drifts. Figure 2 shows STIM calculations of the 211 difference in neutral temperature between the $\mathrm{H}_{3}{ }^{+}$peak altitude and the exobase as a function of 212 latitude. These values are based on the conditions of STIM simulation R15 (see Table 1 of 213 Müller-Wodarg et al., 2012), a representative case for average levels of magnetospheric forcing 214 that reproduces observed auroral temperatures. R15 solar and seasonal conditions are minimum 215 and equinox, respectively. It includes imposed electric fields with peak strength of $76 \mathrm{mV} \mathrm{m}^{-1}$, 216 and allows for a local time averaged auroral particle precipitation flux of $0.62 \mathrm{~mW} \mathrm{~m}^{-2}$ of $10 \mathrm{keV}$

217 electrons. By applying the temperature differential profile of Figure 2 to the exospheric 218 temperature profiles of Figure 1, we can thus derive estimates of $\mathrm{H}_{3}{ }^{+}$temperature as a function of 219 latitude. 


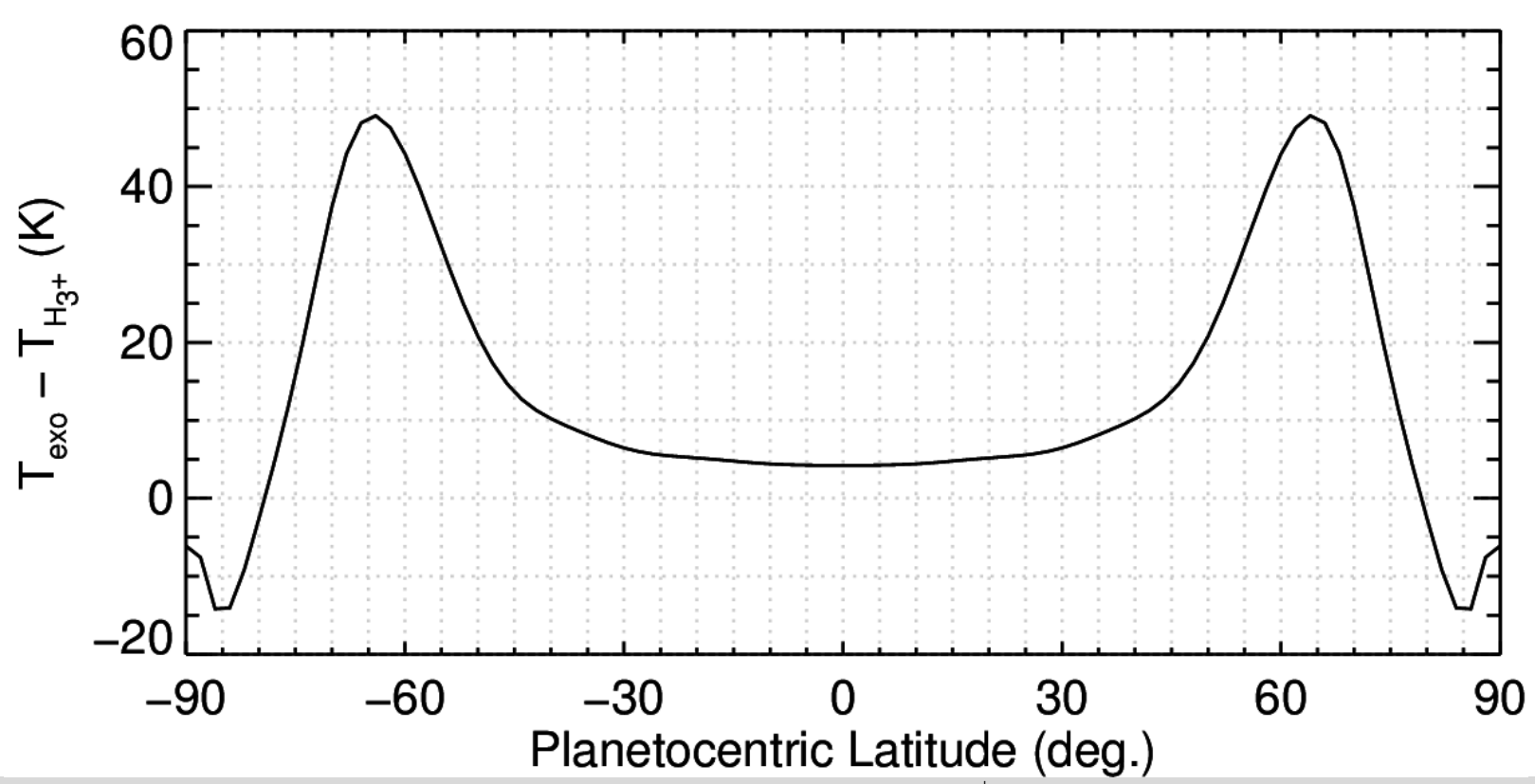

Figure 2. Difference in neutral temperature between the $\mathrm{H}_{3}{ }^{+}$peak altitude and the exobase, as calculated by STIM (Müller-Wodarg et al., 2012).

\subsection{Estimation of $\mathrm{H}_{3}{ }^{+}$Column Densities from Ring Rain Observations}

Under conditions of local thermodynamical equilibrium, the energy emitted by a

223 molecule of $\mathrm{H}_{3}{ }^{+}$during a single ro-vibrational transition is given by (Stallard et al., 2002):

$$
\mathrm{I}_{\text {calc }}^{\text {mol. }}=\frac{\mathrm{g}(2 \mathrm{~J}+1) \mathrm{hc \omega} \text { Aexp }\left[\frac{-\mathrm{E}^{\prime}}{\mathrm{kT}}\right]}{4 \pi \mathrm{Q}(\mathrm{T})}
$$

224 where $\mathrm{I}_{\text {calc }}^{\text {mol. }}$ (in $\mathrm{W} \mathrm{str}{ }^{-1}$ molecule ${ }^{-1}$ ) is the intensity of a given $\mathrm{H}_{3}{ }^{+}$ro-vibrational transition line at

225 temperature $T$ and at wavenumber $\omega$. The nuclear spin degeneracy is given as $g, J$ is the angular

226 momentum of the molecule, $A$ is the Einstein $A$-coefficient for spontaneous emission, $\mathrm{E}^{\prime}$ is the

227 upper energy level of the transition, $h$ and $k$ are the Planck and Boltzmann constants, 228 respectively, and $c$ is the speed of light. $Q(T)$ is the partition function at temperature $T$, for 229 which updated coefficients are given by Miller et al. (2010). These line parameters have been 
230 calculated ab initio by Neale et al. (1996). Multiplying Eq. (1) by the column density of emitting

$231 \mathrm{H}_{3}{ }^{+}, \mathrm{N}_{\mathrm{H}_{3}^{+}}$, yields the total observed flux at wavenumber $\omega$ for constant temperature T:

$$
\mathrm{I}_{o b s}=\mathrm{I}_{c a l c}^{\text {mol. }}(\mathrm{T}) \mathrm{N}_{H_{3}^{+}}
$$

232 This is the value reported by O'Donoghue et al. (2013) in their ring rain observations. First 233 principles calculations, represented here by $I_{\text {calc }}^{\text {mol. }}$, are the means by which $\mathrm{H}_{3}{ }^{+}$column densities 234 can be derived from observed ro-vibrational spectral line intensities. Typically this calculation is 235 performed after the temperatures are first determined from observed spectral line intensity ratios 236 (e.g., Melin et al., 2011). However, as the O'Donoghue et al. detections are too weak to derive 237 temperatures directly, in this work we must use the temperatures estimated in Section 2.2.

238 In order to streamline the process of calculating $N_{H_{3}^{+}}$for 40 different ring rain latitude 239 elements (O'Donoghue et al., 2013) across a wide range of different temperatures, we first 240 develop a parameterization that links $\mathrm{H}_{3}{ }^{+}$column density, temperature and observed intensity. 241 This parameterization comes from previous first principles calculations of $\mathrm{H}_{3}{ }^{+}$emission based on 242 a wide range of STIM-generated atmospheres spanning neutral temperatures from $\sim 400-800 \mathrm{~K}$ 243 (Figure 12 and associated text of Müller-Wodarg et al., 2012), and is tailored for the $\mathrm{Q}\left(1,0^{-}\right)$line 244 at $3.953 \mu \mathrm{m}$. It is given as:

$$
N_{H_{3}^{+}}=\frac{1}{T_{H_{3}^{+}}^{4}} \exp \left\{\frac{\left(\ln I_{H_{3}^{+}}+87.40\right)}{1.167}\right\}
$$

245 where $N_{H_{3}^{+}}$is column density in $\mathrm{m}^{-2}, T_{H_{3}^{+}}$is temperature in $\mathrm{K}$, and $I_{H_{3}^{+}}$is the $\mathrm{Q}\left(1,0^{-}\right)$line 246 intensity in $\mathrm{W} \mathrm{m}^{-2} \mathrm{str}^{-1}$. 


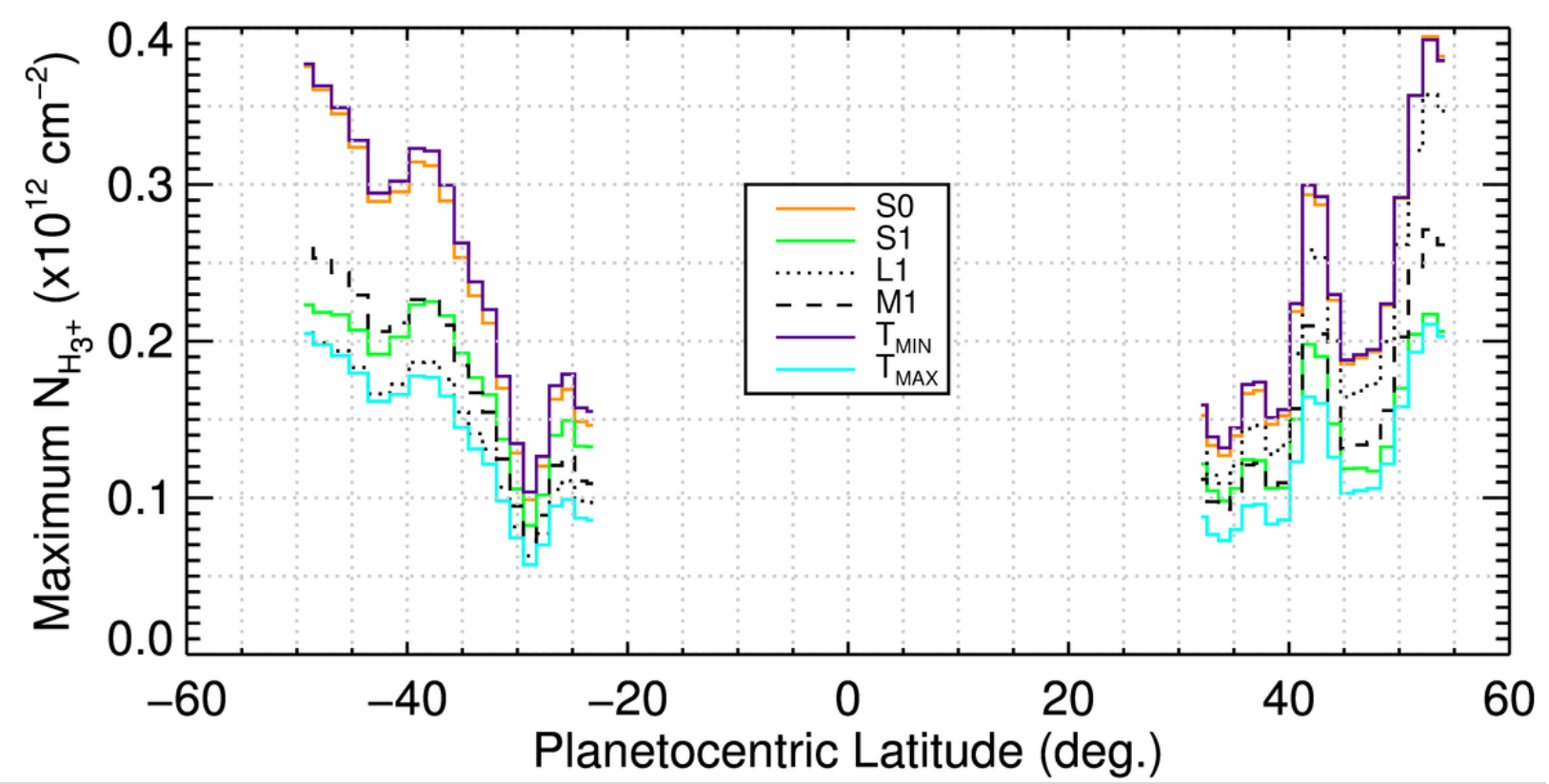

Figure 3. Maximum Saturn ring rain $\mathrm{H}_{3}{ }^{+}$column densities estimated from Eq. (3), using observed $\mathrm{Q}\left(1,0^{-}\right)$spectral intensities and uncertainties at $3.953 \mu \mathrm{m}$ (Figure 2 of $O^{\prime}$ Donoghue et al., 2013), $\mathrm{H}_{3}{ }^{+}$temperature profiles based on observed exospheric temperatures (Figure 1), and modeled temperature differentials (Figure 2). The identifiers in the legend refer to temperature fits from Figure 1.

In combination with the temperature profiles of Figure 1 and Figure 2, and the $\mathrm{Q}\left(1,0^{-}\right)$

248 line intensities shown in Figure 2 of O’Donoghue et al. (2013), Eq. (3) allows a direct 249 calculation of the observed $\mathrm{H}_{3}{ }^{+}$column density as a function of latitude, shown in Figure 3. In 250 order to estimate the maximum $\mathrm{H}_{3}{ }^{+}$column densities, the maximum observed $\mathrm{Q}\left(1,0^{-}\right)$line 251 intensities are used (i.e., full 3-sigma offset from the profile shown in Figure 2 of O'Donoghue et 252 al., 2013). As in Figure 1, the orange and green curves (S0 and S1) assume a smoothly varying 253 global temperature behavior, while the dotted curve (L1) assumes a linear behavior with latitude, 254 and the dashed curve (M1) uses the global mean temperature, constant with latitude. The 255 jaggedness of the profiles shown in Figure 3 represents the latitude resolution in the O'Donoghue 256 et al. (2013) observations. By comparing modeled $\mathrm{H}_{3}{ }^{+}$column densities with those of Figure 3,

257 we will be able to place constraints on possible ring-derived water influxes that could give rise to 258 the observed structure. Higher estimated $\mathrm{H}_{3}{ }^{+}$temperatures correspond to smaller calculated 
259 column densities. The purple and cyan curves in Figure 3 ( $\mathrm{T}_{\mathrm{MIN}}$ and $\mathrm{T}_{\mathrm{MAX}}$ ), therefore, outline a

260 range of minimum and maximum $\mathrm{H}_{3}{ }^{+}$column densities in that any assumed thermospheric

261 temperature profiles that fall within the temperature range indicated in Figure 1 would yield $\mathrm{H}_{3}{ }^{+}$

262 column density estimates between the cyan and purple curves of Figure 3.

263

\section{2.4. Ionospheric Chemistry}

265

In order to match observed electron densities, models must convert $\mathrm{H}^{+}-\mathrm{a}$ dominant and

266 long lived atomic ion in Saturn's ionosphere - into a short lived molecular ion. The two most

267 commonly considered chemical loss pathways for $\mathrm{H}^{+}$are:

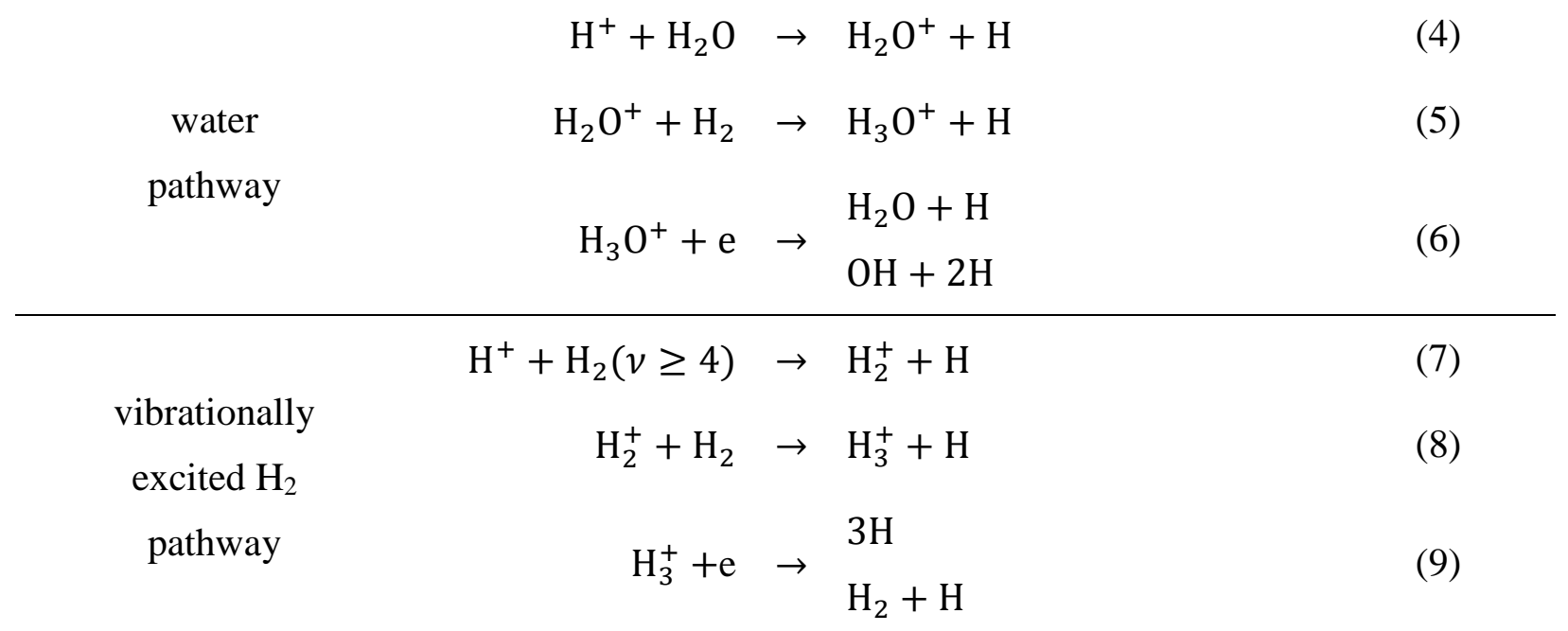

268 While the water-group process of ion and electron removal via reactions (4)-(6) has been

269 discussed already in Section 2.1., it is worth noting that other oxygen bearing compounds (such

270 as $\mathrm{OH}$ ) could perform a similar function, with the effective loss rate being driven by the analog

271 to reaction (4).

272 An alternative method of reducing $\mathrm{H}^{+}$densities, reaction (7), is exothermic only when $\mathrm{H}_{2}$ 273 is excited to the $4^{\text {th }}$ or higher vibrational state (McElroy, 1973). The reaction rate for (7) is

274 thought to be near its maximum kinetic rate (e.g., Huestis, 2008; Huestis et al., 2008); however 
275 the population of vibrationally excited molecular hydrogen, $\mathrm{H}_{2}(v \geq 4)-$ hereafter $\mathbf{H}_{2}{ }^{*}-$ is to date 276 not constrained by observations. Assumed abundances of $\mathrm{H}_{2}{ }^{*}$ vary by over 4 orders of 277 magnitude in model reproductions of radio occultation observations (Majeed et al., 1990, 1991; 278 Majeed and McConnell, 1991; Moses and Bass, 2000). Furthermore, there are only two (related) 279 first principles calculations of $\mathrm{H}_{2}{ }^{*}$ at Saturn (Majeed et al., 1990, 1991). All later ionospheric 280 studies have used various parameterizations that either derived an $\mathrm{H}_{2}{ }^{*}$ distribution based on a 281 single assumed vibrational temperature (Majeed and McConnell, 1991, 1996; see also Cravens, 282 1987), or have modified the calculated distributions of $\mathrm{H}_{2}{ }^{*}$ by Majeed et al. (1991) in some 283 fashion (e.g., Moses and Bass, 2000; Moore et al., 2006, 2010). In short, at present $\mathrm{H}_{2}{ }^{*}$ is an 284 unconstrained parameter at Saturn, and must be treated as such along with the external water 285 influx.

\subsection{Modeling Approach}

For this work we use a 1-D ionospheric module of STIM that solves the ion continuity 289 and momentum equations over a fixed neutral background (Moore et al., 2004, 2008), taken 290 from the 3-D STIM GCM (Müller-Wodarg et al., 2012). This approach is necessary in order to 291 fully explore the possible parameter space of water influxes and $\mathrm{H}_{2}{ }^{*}$ populations with sufficient 292 precision; it would be computationally prohibitive to treat the $>10^{5}$ model runs performed here in 293 full 3-D. However, this approach is also justified by the goal of performing $\mathrm{H}_{3}{ }^{+}$model-data 294 column density comparisons, as the short chemical lifetimes for $\mathrm{H}_{3}{ }^{+}$ions at Saturn mean they are 295 firmly in the photochemical regime, and therefore relatively insensitive to global dynamics. In 296 order to generate neutral atmospheric profiles that correlate with the temperatures described in 297 Section 2.1., temperatures are fixed at $\sim 135 \mathrm{~K}$ at the base of the thermosphere, and then scaled to 
298 the exospheric temperatures derived in Figure 1 while retaining the qualitative vertical thermal 299 structure calculated within STIM. In similar fashion, neutral densities are fixed at the 300 homopause and then scaled vertically using scale heights appropriate for the observed 301 temperatures (and using the latitudinal temperature profiles of Figure 1). Water density altitude 302 profiles are based on time-dependent diffusion calculations that include constant topside water 303 influx as a boundary condition (Moore et al., 2006; Moore and Mendillo, 2007). Model calculations rely on solar EUV and X-ray fluxes specified using measurements 305 from the Thermosphere Ionosphere Mesosphere Energetics and Dynamics Solar EUV 306 experiment (TIMED/SEE: Woods et al., 2000, 2005; Woods, 2008). Solar fluxes have been 307 extrapolated to Saturn for 17 April 2011, the date of the ring rain observations (O'Donoghue et 308 al., 2013), with a corresponding F10.7 radio flux index of 114.4, and a sub-solar latitude at 309 Saturn of $9.1^{\circ}$

310 In order to account for the uncertain water influxes and $\mathrm{H}_{2}{ }^{*}$ distributions, we explore a 311 wide range of each in order to find the combination of parameters that best reproduces the $\mathrm{H}_{3}{ }^{+}$ 312 column densities derived from ring rain observations (Figure 3). A sample of such a series of 313 model results is shown in Figure 4, which gives the calculated $\mathrm{H}_{3}{ }^{+}$column density at noon - the 314 local time of ring rain observations - as a function of water influx, $\Phi_{\mathrm{H}_{2} \mathrm{O}}$, and $\mathrm{H}_{2}{ }^{*}$ population, 315 represented here as $k_{f a c}$. (As the model solves ion continuity and momentum equations via 316 explicit time integration, results are available for all local times, though only noon values are 317 shown unless otherwise specified.) The effective reaction rate for (7), $k_{\text {eff, }}$ is taken to be a 318 multiplicative factor $\left(k_{f a c}\right)$ of the Moses and Bass (2000) rate, $k_{M B 2000}$ (see also Majeed et al., 319 1991), and represents a combination of the chemical reaction rate, $k_{7}$, and the fraction of 320 vibrationally excited $\mathrm{H}_{2}, \mathrm{H}_{2}(v \geq 4) / \mathrm{H}_{2}$. Similar model simulations are undertaken for all 40 of the 
322 zenith angles and neutral background atmospheres.

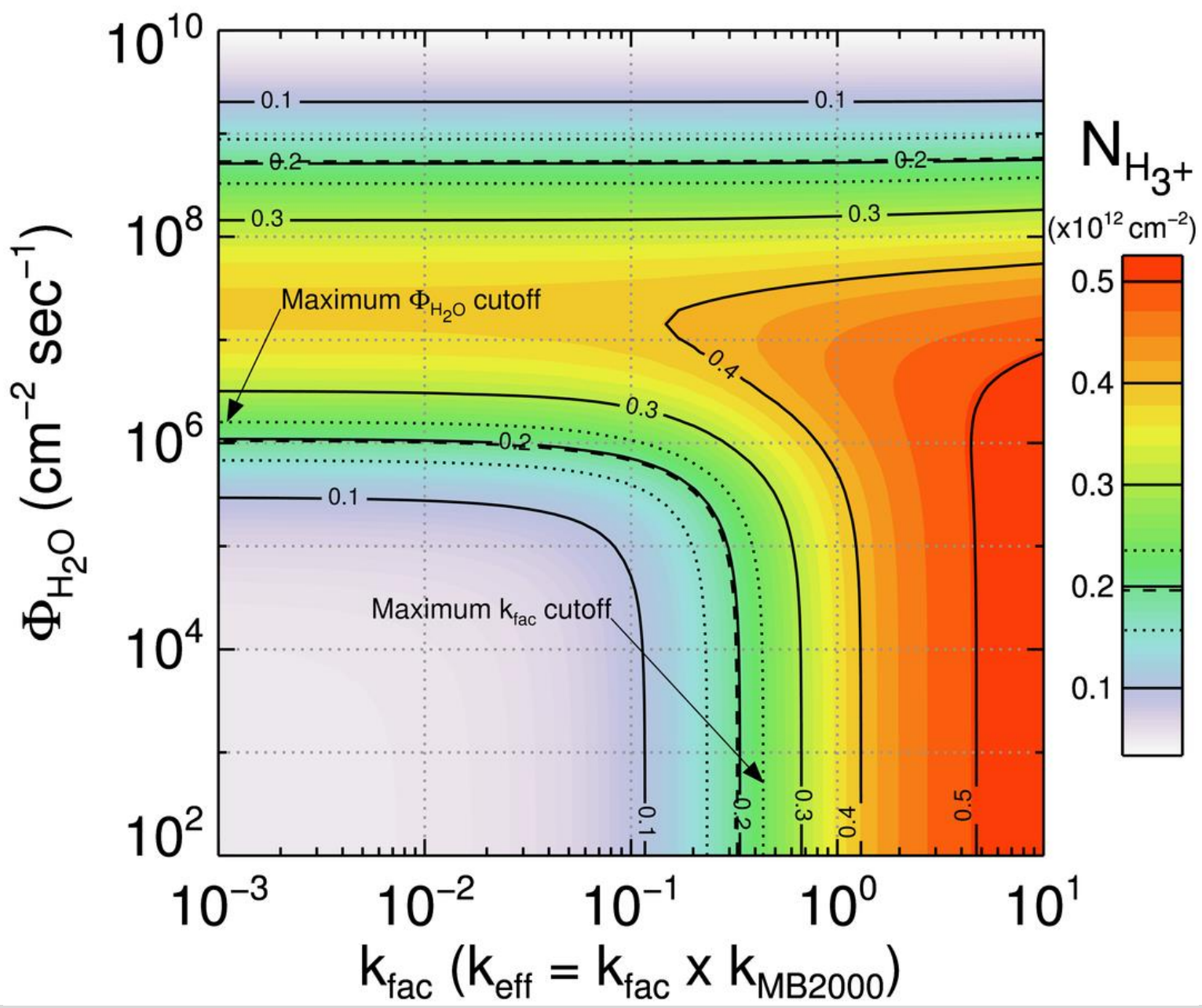

Figure 4. Contours of modeled $\mathrm{H}_{3}{ }^{+}$column densities for a range of water influxes, $\Phi_{\mathrm{H}_{2} \mathrm{O}}$, and populations of vibrationally excited $\mathrm{H}_{2}$, represented here by $k_{f a c}$ (see text). Calculation results are for $-35^{\circ}$ latitude and a solar local time of noon. The dashed curves indicate the $\mathrm{H}_{3}{ }^{+}$column density derived from ring rain observations at $-35^{\circ}$ latitude, based on the green profile (S1) from Figure 3, and therefore identify the combinations of $\Phi_{\mathrm{H}_{2} \mathrm{O}}$ and $k_{f a c}$ parameters that can reproduce the observation at that latitude. Dotted curves outline the range of estimated $\mathrm{H}_{3}{ }^{+}$column densities that result from accounting for a 3-sigma uncertainty in observed $\mathrm{H}_{3}{ }^{+}$emission intensity (i.e., Figure 2 of O'Donoghue et al., 2013). Maximum $\Phi_{\mathrm{H}_{2} \mathrm{O}}$ and $k_{f a c}$ cutoffs are indicated by arrows (see text).

There are two important patterns to note in Figure 4. First, for water influxes above $\sim 10^{5}$ $\mathrm{cm}^{-2} \mathrm{sec}^{-1}$, calculated $\mathrm{H}_{3}{ }^{+}$column densities initially increase and then eventually decrease as

325 more and more water is introduced into Saturn's ionosphere (above $\sim 2 \times 10^{7} \mathrm{~cm}^{-2} \mathrm{sec}^{-1}$ ). In other 
words, water can act as both a "source" and a sink for $\mathrm{H}_{3}{ }^{+}$. This apparent anomaly is easily

327 explained by the fact that the dissociative recombination rate of $\mathrm{H}_{3}{ }^{+}$is relatively fast and

328 therefore a reduction in modeled electron densities, such as the one resulting from the $\mathrm{H}^{+}+\mathrm{H}_{2} \mathrm{O}$

329 charge exchange reaction chain (4)-(6), leads to a corresponding reduction in the $\mathrm{H}_{3}^{+}+\mathrm{e}^{-}$loss

330 process. In addition, for most values of $k_{f a c}$, there is a maximum modeled $\mathrm{H}_{3}{ }^{+}$column density for

331 a water influx of $\sim 2 \times 10^{7} \mathrm{~cm}^{-2} \mathrm{sec}^{-1}$. Second, an increase in $k_{f a c}$ represents an increase in the

332 effective rate for the $\mathrm{H}^{+}+\mathrm{H}_{2}(v \geq 4)$ reaction, and hence an increase in the production of $\mathrm{H}_{3}{ }^{+}$via

333 reaction (8). Consequently modeled $\mathrm{H}_{3}{ }^{+}$column densities are always larger than observed

334 densities for $k_{f a c}$ values of $\sim 1$ or more and water influxes of $\sim 10^{7} \mathrm{~cm}^{-2} \mathrm{sec}^{-1}$ or less. At extremely

335 large values of external water influx, above $10^{8} \mathrm{~cm}^{-2} \mathrm{sec}^{-1}$, there is insufficient $\mathrm{H}^{+}$for Reaction

336 (7) to play any further significant role, and water chemistry dominates calculated $\mathrm{H}_{3}{ }^{+}$densities.

337 Figure 5 presents model results for the same range of parameters explored in Figure 4, 338 except it shows electron column densities at dawn rather than $\mathrm{H}_{3}{ }^{+}$column densities at noon. In

339 this way the measured electron column density from Cassini radio occultation 047x (Table 1 of

340 Kliore et al., 2009) - indicated by the dashed curve - can be compared with the model results.

341 Occultation $047 \mathrm{x}$ is chosen as a typical representative of the 5 total Cassini radio occultations at 342 ring rain latitudes $\left(047 \mathrm{x}, 051 \mathrm{n}, 051 \mathrm{x}, 070 \mathrm{n}\right.$, and 072n), as it is at approximately $-35^{\circ}$ 343 planetocentric latitude, and therefore can easily be compared with the conditions of Figure 4 . It

344 should be noted that, while radio occultations sample a range of latitudes - e.g., $36.7 \mathrm{~S}$ to $41.2 \mathrm{~S}$

345 planetographic latitude for $047 \mathrm{x}$ - the majority of the electron column content associated with a

346 particular occultation is generated in the lower ionosphere, near the electron density peak, and

347 therefore near the end of the quoted latitude range (e.g., 41.2S for 047x). Finally, it is most

348 likely a coincidence that similar combinations of $\Phi_{\mathrm{H}_{2} \mathrm{O}}$ and $k_{f a c}$ appear capable of reproducing the 
350 observations were made under very different seasonal and solar conditions.

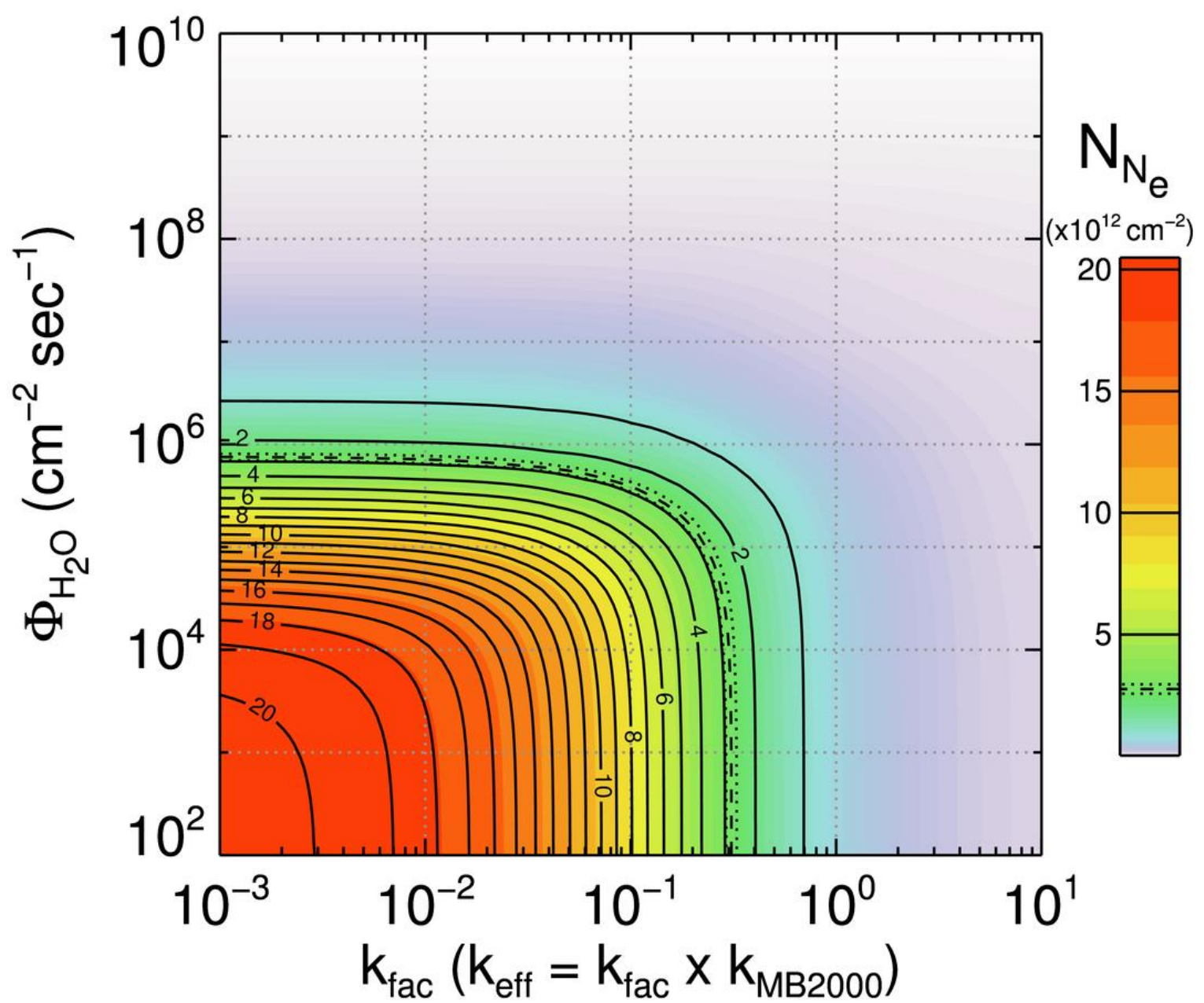

Figure 5. Contours of modeled electron column densities for a range of water influxes, $\Phi_{\mathrm{H}_{2} \mathrm{O}}$, and populations of vibrationally excited $\mathrm{H}_{2}$, represented here by $k_{\text {fac }}$ (see text). Calculation results are for $-35^{\circ}$ latitude and a solar local time of dawn. The dashed curve indicates the electron column density derived from Cassini radio occultation observation 047x at $-41.2^{\circ}$ planetographic latitude (Kliore et al., 2009; Moore et al., 2010), or approximately $-35^{\circ}$ planetocentric latitude. Dotted curves represent a possible 3-sigma range of observed electron column densities, determined by applying the maximum uncertainty quoted Nagy et al. (2006), as no uncertainties are reported in Kliore et al. (2009).

Both Figure 4 and Figure 5 reveal an interesting behavior: for small values of $\Phi_{\mathrm{H}_{2} \mathrm{O}}$,

352 modeled column densities increase $\left(\mathrm{H}_{3}{ }^{+}\right)$or decrease $\left(\mathrm{N}_{\mathrm{e}}\right)$ monotonically with increasing $k_{f a c}$. A

353 similar behavior is found for small values of $k_{f a c}$, with the exception that modeled $\mathrm{H}_{3}{ }^{+}$column

354 densities maximize near $2 \times 10^{7} \mathrm{~cm}^{-2} \mathrm{sec}^{-1}$, and then decrease again for larger water influxes. In 
355 other words, based solely on Figure 4, there are two families of $\Phi_{\mathrm{H}_{2} \mathrm{O}}$ and $k_{f a c}$ values that are 356 capable of reproducing observed $\mathrm{H}_{3}{ }^{+}$column densities, a "low" water influx solution $\left(\Phi_{\mathrm{H}_{2} \mathrm{O}}<\right.$ $\left.35710^{7} \mathrm{~cm}^{-2} \mathrm{sec}^{-1}\right)$ and "high" water influx solution $\left(\Phi_{\mathrm{H}_{2} \mathrm{O}}>10^{8} \mathrm{~cm}^{-2} \mathrm{sec}^{-1}\right)$. By making use of the 358 model results of Figure 5, however, we can exclude the high water influx solution, as the 359 modeled electron densities there are far too small when compared with radio occultation 360 measurements. The electron column densities from the 5 Cassini radio occultations that overlap 361 with ring rain latitudes $-047 \mathrm{x}, 051 \mathrm{n}, 051 \mathrm{x}, 070 \mathrm{n}$, and $072 \mathrm{n}-$ are $2.76,1.76,2.41,1.66$, and 1.64, 362 respectively (in units of $10^{12} \mathrm{~cm}^{-2}$; Kliore et al., 2009; Moore et al., 2010). All of these values lie 363 within the outermost solid contour of Figure 5.

364 Therefore, by focusing on model comparisons with the $\mathrm{H}_{3}{ }^{+}$column densities from Figure 3653 , and by using Figure 5 to discount "high" water influx solutions, we can then derive a 366 constraint on the maximum values for water influx and $k_{f a c}$ as a function of latitude. For 367 example, in Figure 4, there are many combinations of $\Phi_{\mathrm{H}_{2} \mathrm{O}}$ and $k_{f a c}$ that are capable of 368 reproducing the $\sim 0.2 \times 10^{12} \mathrm{~cm}^{-2} \mathrm{H}_{3}{ }^{+}$column density derived from ring rain observations, but 369 there are clear cutoffs in the maximum allowable water influx $\left(\sim 10^{6} \mathrm{~cm}^{-2} \mathrm{sec}^{-1}\right)$ and $k_{f a c}(\sim 0.3)$ 370 values.

\section{3. Results}

373 3.1. Maximum Ring-Derived Water Influx vs. Latitude

374 Results throughout the remainder of the text are based on simulations that explore 60

$375 \Phi_{\mathrm{H}_{2} \mathrm{O}}$ elements spread evenly in log space across $3 \times 10^{3}-3 \times 10^{7} \mathrm{~cm}^{-2} \mathrm{sec}^{-1}$ and $60 k_{f a c}$ elements 376 spread evenly in log space across 0.003-3. Thus, there are 3600 individual model runs conducted 377 for each of the 40 ring rain latitude elements. Both the $\Phi_{\mathrm{H}_{2} \mathrm{O}}$ and the $k_{f a c}$ ranges are chosen to 
378 capture the full range of possible maximum values for each parameter based on comparisons 379 with $\mathrm{H}_{3}{ }^{+}$column densities (Figure 4).

380 Figure 6 presents maximum ring-derived water influxes versus latitude, determined from 381 model comparisons with the $\mathrm{H}_{3}{ }^{+}$column densities shown in Figure 3. The colored, dotted and 382 dashed profiles correspond to those given Figure 3, and therefore represent a range of possible 383 upper limits on water influx, based on the various assumptions regarding $\mathrm{H}_{3}{ }^{+}$temperatures at 384 ring rain latitudes. In general, maximum water influxes are found to be larger in the southern 385 hemisphere, while there are local maxima near $38^{\circ}$ and $48^{\circ}$ in the south and $42^{\circ}$ and $53^{\circ}$ in the 386 north. The "instability region" described by O'Donoghue et al. (2013) maps to approximately $387 \quad 36-39^{\circ}$ latitude in the south and $42-45^{\circ}$ latitude in the north. This region lies between two 388 "instability radii" located in the rings near $1.52 \mathrm{R}_{\mathrm{S}}$ and $1.62 \mathrm{R}_{\mathrm{S}}$, respectively. The former 389 represents a stability limit for highly charged particles launched azimuthally in Saturn's ring 390 plane at Keplerian circular velocity, while the latter represents a stability limit for particles in 391 circular orbit (Northrop and Hill, 1982, 1983). At these radii outward centrifugal forces on 392 charged particles are balanced by inward gravitational forces, leading to unstable regions in 393 which particles can easily flow into Saturn's atmosphere along magnetic field lines. Theory 394 therefore predicts an enhancement of influx into Saturn's atmosphere at the latitudes on either 395 side of the instability region. Though there remains significant scatter, on average Figure 6 does 396 have local maxima in the derived water influxes at the instability latitudes. The overall trend in 397 water influx with latitude, however, differs from previous results (e.g., Moore et al., 2010), a fact 398 discussed in more detail in Section 4.1. 




Figure 6. Maximum ring rain water influx estimates versus latitude. A series of model simulations exploring a 60x60 grid of $\Phi_{\mathrm{H}_{2} \mathrm{O}}$ and $k_{f a c}$ values (see text) is used to find the maximum water influx that can reproduce the observed $\mathrm{H}_{3}{ }^{+}$column densities derived in Figure 3. Each profile represents the results from a different assumption regarding $\mathrm{H}_{3}{ }^{+}$temperatures at ring rain latitudes (see Figure 1).

Figure 7 shows the maximum derived $k_{f a c}$ values versus latitude, based on model comparisons with the $\mathrm{H}_{3}{ }^{+}$column densities derived in Figure 3. Rather than showing the results 402 of each of the six different assumptions made regarding ring rain temperatures (see Figure 1), 403 Figure 7 is simplified and shows only results from the $\mathrm{S} 1$ fit (i.e., using the measurements from 404 Smith et al., 1983; Koskinen et al., 2013; Vervack and Moses, 2013). In addition, the mean 405 hemispheric maximum $k_{f a c}$ values are also indicated by the dashed lines: 0.26 in the south and 4060.17 in the north. Previous STIM comparisons with Cassini radio occultation measurements 407 favored $k_{f a c}$ values of 0.075-0.25 (Moore et al., 2006) and 0.125 (Moore et al., 2010), a similar 408 range to that derived in Figure 7. Improved knowledge regarding the true values for $k_{f a c}$ present 409 in Saturn's atmosphere could be used to refine the estimates for water influx. Rather than 410 finding a maximum ring rain water influx for any value of $k_{f a c}$, as in Figure 6, we could find the 
411 model simulation that is best able to reproduce the observed $\mathrm{H}_{3}{ }^{+}$column densities along a 412 specified track of $k_{f a c}$ values. For example, if $k_{f a c}$ were fixed to 0.125 , then the resulting best fit 413 water influxes would be a factor of 2-3 smaller on average than the maximum values shown in 414 Figure 6. For $k_{f a c}=0.25$ the water influxes would be reduced by a factor of 10-12 on average.

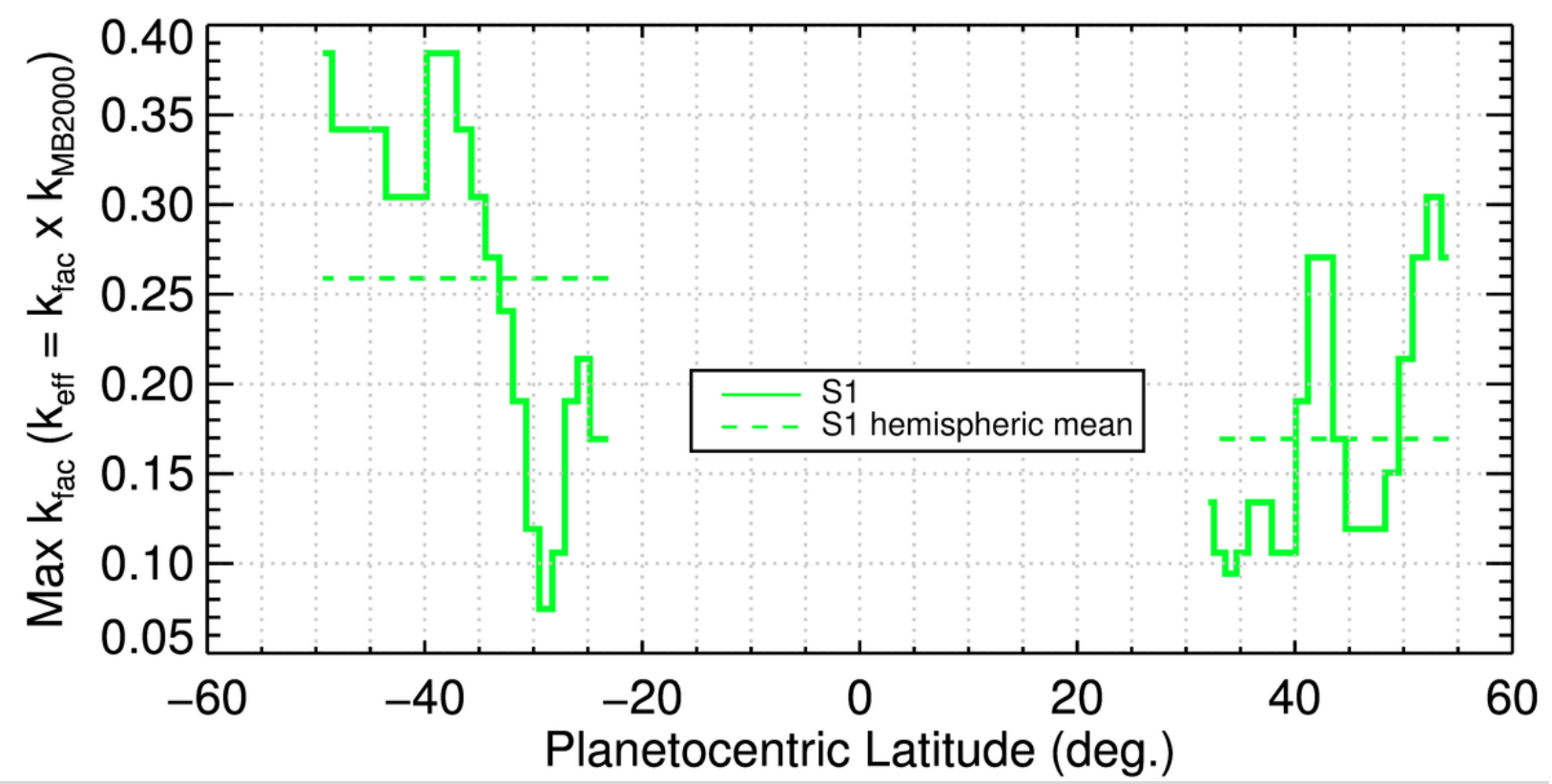

Figure 7. Same as Figure 6, but for $k_{f a c}$ values. Additionally, rather than show the $k_{f a c}$ values derived for each assumed $\mathrm{H}_{3}{ }^{+}$temperature profile separately, we only show the $k_{f a c}$ values resulting from the green profile in Figure 3 (solid line) along with the mean $k_{f a c}$ value for each hemisphere (dashed line).

Based on UV spectra of Saturn obtained in 1994 with the Hubble Space Telescope

417 (HST), Prangé et al. (2006) tentatively attributed an enhancement in the contrast in the $33^{\circ} \mathrm{S}$

418 planetocentric (R. Prangé, personal communication) latitude spectrum at $2000 \AA$ and $1720 \AA$ to a

419 locally enhanced water abundance. While there is no obvious corresponding feature near $33^{\circ} \mathrm{S}$

420 latitude in Figure 6, pointing uncertainties in the Prangé et al. measurements translate to an $8^{\circ}$

421 uncertainty in latitude. Two local maxima in water influx derived in Figure 6 are within this $8^{\circ}$

422 latitude window, one near $26^{\circ} \mathrm{S}$ and one near $38^{\circ} \mathrm{S}$. Prangé et al. also observed at $41_{-11^{\circ}}^{\circ} \mathrm{S}$ and

$42352_{-12^{\circ}}^{\circ} S$ latitude, but were only able to place upper limits on water column density at those 
424 latitudes (of $<1.4 \times 10^{16} \mathrm{~cm}^{-2}$ and $<3.4 \times 10^{16} \mathrm{~cm}^{-2}$, respectively). However, Prangé et al. noted

425 that a local minimum in hydrocarbon abundance was present at $41^{\circ} \mathrm{S}$, which could be interpreted

426 as indirect evidence for a locally enhanced water influx, based on the modeling of Moses et al.

427 (2000). Therefore, while there is no obvious direct correspondence between the possible 428 detection of locally enhanced water abundance by Prangé et al. and by the latitudinal variations 429 in water influx derived here, they share some consistent characteristics.

\subsection{Global Water Influx \& Exogenous Water Sources}

432 Water influxes derived here can be combined with previous results in order to estimate a 433 global water influx. STIM comparisons with Cassini radio occultations were best able to 434 reproduce the measured electron densities in Saturn's ionosphere when a latitudinally varying 435 water influx was considered (Moore et al., 2010). Specifically, Moore et al. used a Gaussian 436 water influx profile that peaked at Saturn's equator and had a variance $\sigma$ of $10^{\circ}$. Figure 8 shows 437 this water profile (black, dashed line) along with the maximum water influxes from the green 438 solid curve in Figure 6, and with water influx estimates using a fixed $k_{f a c}$ of 0.125 and the S1 439 temperature fit (green, dotted line). The combined maximum water influx profile (i.e., solid + 440 dashed curves) leads to an average water influx of $1.4 \times 10^{6} \mathrm{H}_{2} \mathrm{O}$ molecules $\mathrm{cm}^{-2} \mathrm{sec}^{-1}$ (averaged 441 over the entire oblate Saturn spheroid) and a total global influx of 5.9x $10^{26} \mathrm{H}_{2} \mathrm{O}$ molecules $\sec ^{-1}$. 442 This average influx is fairly close to the globally averaged value of $\sim 1.5 \times 10^{6} \mathrm{~cm}^{-2} \mathrm{sec}^{-1}$ derived 443 by Moses et al. (2000) based on ISO observations (Feuchtgruber et al., 1997). While the 444 variation with latitude shown in Figure 8 is not strictly supported by Moses et al. (2000), as they 445 found a best match to ISO observations using a constant influx versus latitude, it is not 446 necessarily prohibited either. They only explored enhancements of $10^{7}$ and $10^{8}$ molecules $\mathrm{cm}^{-2}$ 
$447 \mathrm{sec}^{-1}$, above the range of the influxes calculated here. Therefore, it is possible that the less 448 drastic variations in influx with latitudinal shown in Figure 8 are consistent with ISO 449 observations.

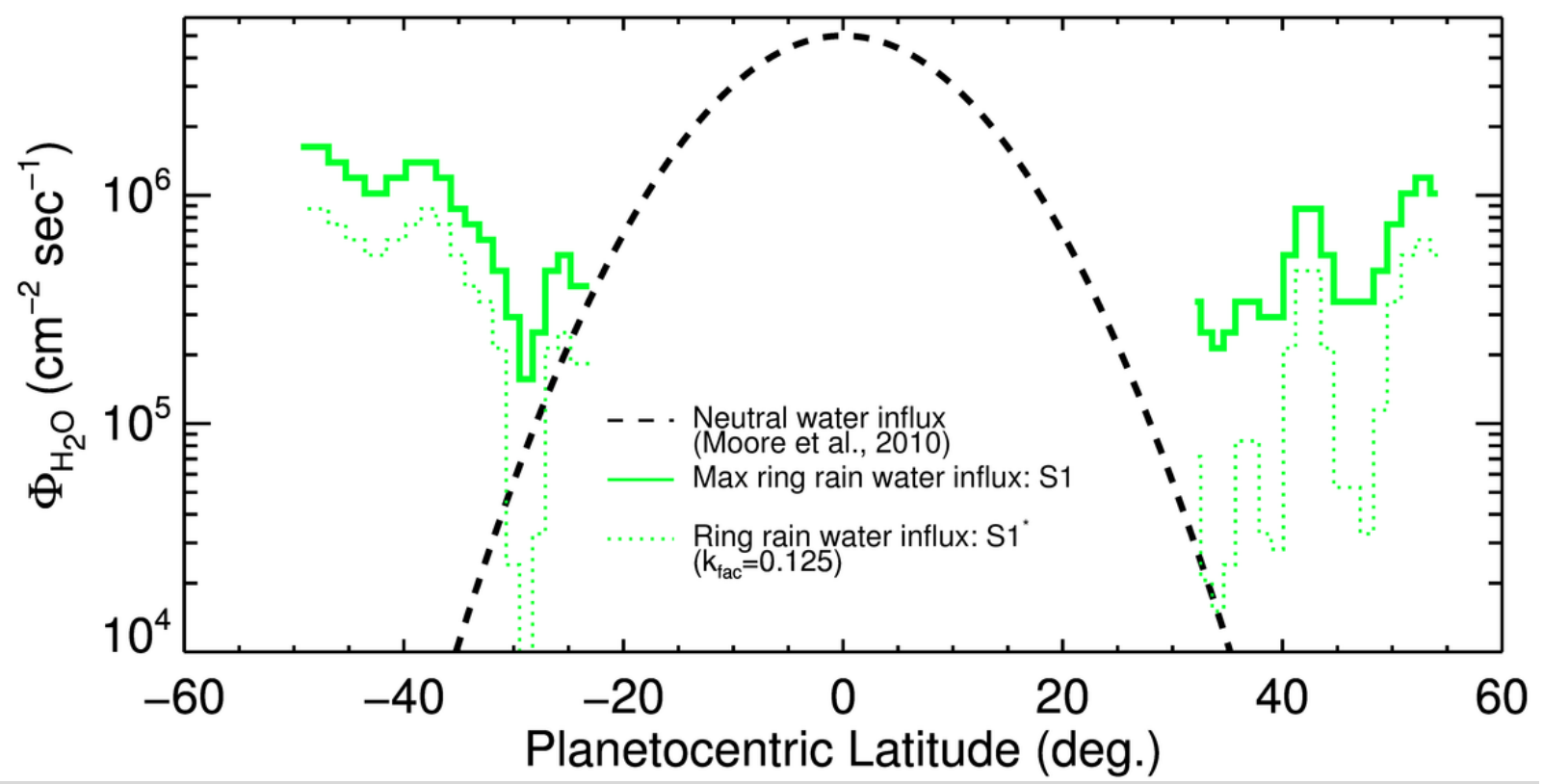

Figure 8. Estimates of global water influxes based on the maximum water influxes calculated from ring rain observations (solid green profile, Figure 6), water influxes estimated using a fixed $k_{f a c}$ of 0.125 and the S1 temperature fit (dotted green profile), and water influxes derived from model comparisons with Cassini radio occultation measurements (dashed black curve, Moore et al., 2010).

Global water influx values given above are computed using the maximum water influxes

451 shown in Figure 8 (green solid profile). If $k_{f a c}$ is instead fixed to a specific value, such as 0.125

452 (e.g., Moore et al., 2010), then the resulting water influx values are reduced by a factor of 2-3 on

453 average, yielding a globally averaged influx of $1.3 \times 10^{6} \mathrm{H}_{2} \mathrm{O}$ molecules $\mathrm{cm}^{-2} \mathrm{sec}^{-1}$ and a total

454 global influx of $5.4 \times 10^{26} \mathrm{sec}^{-1}$. Different assumptions regarding temperatures at ring rain

455 latitudes also affect the computed global influx values. Larger temperatures correspond to

456 smaller $\mathrm{H}_{3}{ }^{+}$densities (Figure 3), which in turn reduce the water influx estimates (Figure 6). The

457 full range of globally averaged maximum water influxes, based on the temperature profiles of

458 Figure 1 and the resulting maximum water influxes of Figure 6, is $(1.3-2.3) \times 10^{6} \mathrm{~cm}^{-2} \mathrm{sec}^{-1}$. 
459 Similarly, the full range of total global maximum influx is (5.6-10) $\times 10^{26} \mathrm{sec}^{-1}$. Note that the 460 preceding values have been calculated by combining the dashed curve in Figure 8 with model 461 simulation results (Figure 6 and Figure 8). Results which omit the contribution from the dashed 462 curve to the global mean influx $-1.1 \times 10^{6} \mathrm{~cm}^{-2} \mathrm{sec}^{-1}$ and $4.9 \times 10^{26} \mathrm{sec}^{-1}$, respectively - are 463 summarized in Table 1.

464 Table 1. Estimated water influxes from model simulation comparisons with derived ring rain $\mathrm{H}_{3}{ }^{+}$column densities.

465

466

467

468

469

470

471

472

473

474

475

476

\section{Temperature} Fit ID
S0

$\mathrm{S} 1$

L1

M1

$\mathrm{T}_{\text {MIN }}$

$\mathrm{T}_{\mathrm{MAX}}$
Fit Type

\section{Globally Averaged} "Ring Rain" Influx $\left(\times 10^{6} \mathrm{~cm}^{-2} \mathrm{sec}^{-1}\right)$

\section{Total "Ring} Rain" Influx $\left(\mathrm{x10}^{26} \mathrm{sec}^{-1}\right)$

\section{Maximum Water Influx Estimate Method}

sinusoidal [1],[5] 1.2

$$
\text { sinusoidal }
$$

[1],[5],[6]

[1],[5],[6]

0.24

0.31

[1],[5],[6]

0.28

$\mathrm{T}_{\text {exo }}$ minimum

1.2

0.16

5.1

1.0

1.4

1.2

5.2

6.8

Fixed $k_{f a c}$ Water Influx Estimate Method

$\begin{array}{lllll}\mathrm{S}^{*} & \text { sinusoidal; } k_{\text {fac }}=0.125 & {[1],[5],[6]} & 0.11 & 0.49\end{array}$

$\mathrm{S}^{* *} \quad$ sinusoidal; $k_{f a c}=0.25 \quad[1],[5],[6]$

0.04

0.17

${ }^{\mathrm{a}}$ [1] - Smith et al. (1983); [5] - Vervack and Moses (2013); [6] - Koskinen et al. (2013).

While we cannot explicitly identify the source of exogenous water based on the modeling, the derived water influx rates can be further broken into a "neutral" source (Moore et $a l ., 2010$ ) and a "ring rain" ionized source (this work). The total global influx of $5.9 \times 10^{26} \sec ^{-1}$ given above then represents the sum of a neutral source of $4.9 \times 10^{26} \mathrm{sec}^{-1}$ and a ring rain source of $1.0 \times 10^{26} \mathrm{sec}^{-1}$ (S1 temperature fit). Assuming the "neutral" source originates from Enceladus with a rate of $10^{28} \sec ^{-1}$ (Jurac and Richardson, 2007; Cassidy and Johnson, 2010), our derived neutral influx represents $\sim 5 \%$ of the water ejected from Enceladus' plumes, within a factor of two of current model predictions for Enceladus oxygen products lost to Saturn's atmosphere: 10\%, 7\%, 3\%, and 6\%, respectively (Jurac and Richardson, 2007; Cassidy and Johnson, 2010;

Hartogh et al., 2011; Fleshman et al., 2012). Similarly, if the assumed "ring rain" source is entirely from the rings, then it represents about $10 \%$ of the $\sim 10^{27} \mathrm{sec}^{-1}$ ions produced in Saturn's 
477 ring atmosphere (Johnson et al., 2006), and the globally averaged maximum ring-derived water 478 influx of $2.4 \times 10^{5} \mathrm{~cm}^{-2} \mathrm{sec}^{-1}$ is in rough agreement with the total oxygen influx of $\sim 10^{5} \mathrm{~cm}^{-2} \mathrm{sec}^{-1}$ 479 estimated from ring atmosphere models (e.g., Tseng et al., 2010). Furthermore, as first predicted 480 by Connerney (1986), precipitation is expected to be enhanced at southern latitudes due to 481 Saturn's effectively offset magnetic dipole (Burton et al., 2010), independent of Saturn season 482 (e.g., Northrop and Connerney, 1987; Luhmann et al., 2006; Tseng et al., 2010). This 483 enhancement is also present here, though the magnitude is weaker than predicted by a factor of $484 \sim 5$. For example, the mean water influx for $k_{f a c}=0.125$ model results is $4.5 \times 10^{5} \mathrm{~cm}^{-2} \mathrm{sec}^{-1}$ in the 485 southern hemisphere and $2.0 \times 10^{5} \mathrm{~cm}^{-2} \mathrm{sec}^{-1}$ in the northern hemisphere $\left(\mathrm{S} 1^{*}\right.$ temperature fit; 486 green dotted profile in Figure 8). Taken together, these comparisons provide evidence that the 487 oxygen influx at Saturn can be quantitatively attributed to two separate sources: Saturn's rings 488 and Enceladus. Further sources, such as interplanetary dust particles and cometary impacts, 489 remain possible, though are likely not required to explain current observations of Saturn's upper 490 atmosphere.

492 3.3. Ring Mass Loss Rates and Lifetime Estimate

493 In order to estimate the ring mass loss implied by water influxes calculated in this work, 494 we first convert the variations of influx in latitude to variations in radius in Saturn's ring plane. 495 We use the axisymmetric magnetic mapping model of Bunce et al. (2008) with updated internal 496 field coefficients based on Cassini measurements (Burton et al., 2010). We set the height of the 497 ionosphere to $1,100 \mathrm{~km}$ above the 1-bar level, where the peak $\mathrm{H}_{3}{ }^{+}$density is approximately 498 located (Stallard et al., 2012), and use the IAU Saturn equatorial radius value of $60,268 \mathrm{~km}$ 
(Seidelmann et al., 2007). Further details and magnetic mapping model comparisons are given

500 in the O'Donoghue et al. (2013) supplementary information.

$501 \quad$ Figure 9 shows the result of mapping the ring rain water influxes of Figure 8 along 502 magnetic field lines into Saturn's equatorial plane. In order to give some sense of ring structure, 503 we also plot ring normal optical depths measured in the IR from ground based occultations of 28 504 Sgr (Nicholson et al., 2000). Two points are immediately evident from the figure. First, there is 505 a local maximum of ring rain influx at $\sim 1.52 \mathrm{R}_{\mathrm{S}}$, near the edge of the instability radius, consistent 506 with predictions (e.g., Northrop and Hill, 1983). Second, the north-south asymmetry in derived 507 water influxes is even more obvious when plotted together; the southern water fluxes dominate 508 all of the equatorial structure. This last point further strengthens the prediction from ring 509 atmosphere models that the southern ring atmosphere (and resulting precipitation into Saturn's 510 atmosphere) is always stronger than the northern ring atmosphere due to the effectively offset 511 magnetic dipole (e.g., Connerney, 1986; Northrop and Connerney, 1987; Tseng et al., 2010), as 512 the sub-solar point during ring rain observations was $+9.1^{\circ}$, and therefore the sun primarily 513 illuminated the northern ring face.

514 It is difficult to make accurate measurements of the total mass of Saturn's rings, as the 515 standard assumption of uniform distribution of ring particles neglects the possibility of particles 516 clumping into large gravitational aggregates, and therefore previous determinations likely 517 represent only lower bounds on ring mass. Similarly, while we express ring derived precipitation 518 influxes as "water" in this work, they could be equally well represented by heavier oxygen 519 compounds and/or dust grains. Therefore, an estimate of ring mass loss based on the assumption 520 of $\mathrm{H}_{2} \mathrm{O}$ influx is a lower limit. Nevertheless, it is worthwhile to assess the possible impact of our 521 calculated influxes on ring evolution. 


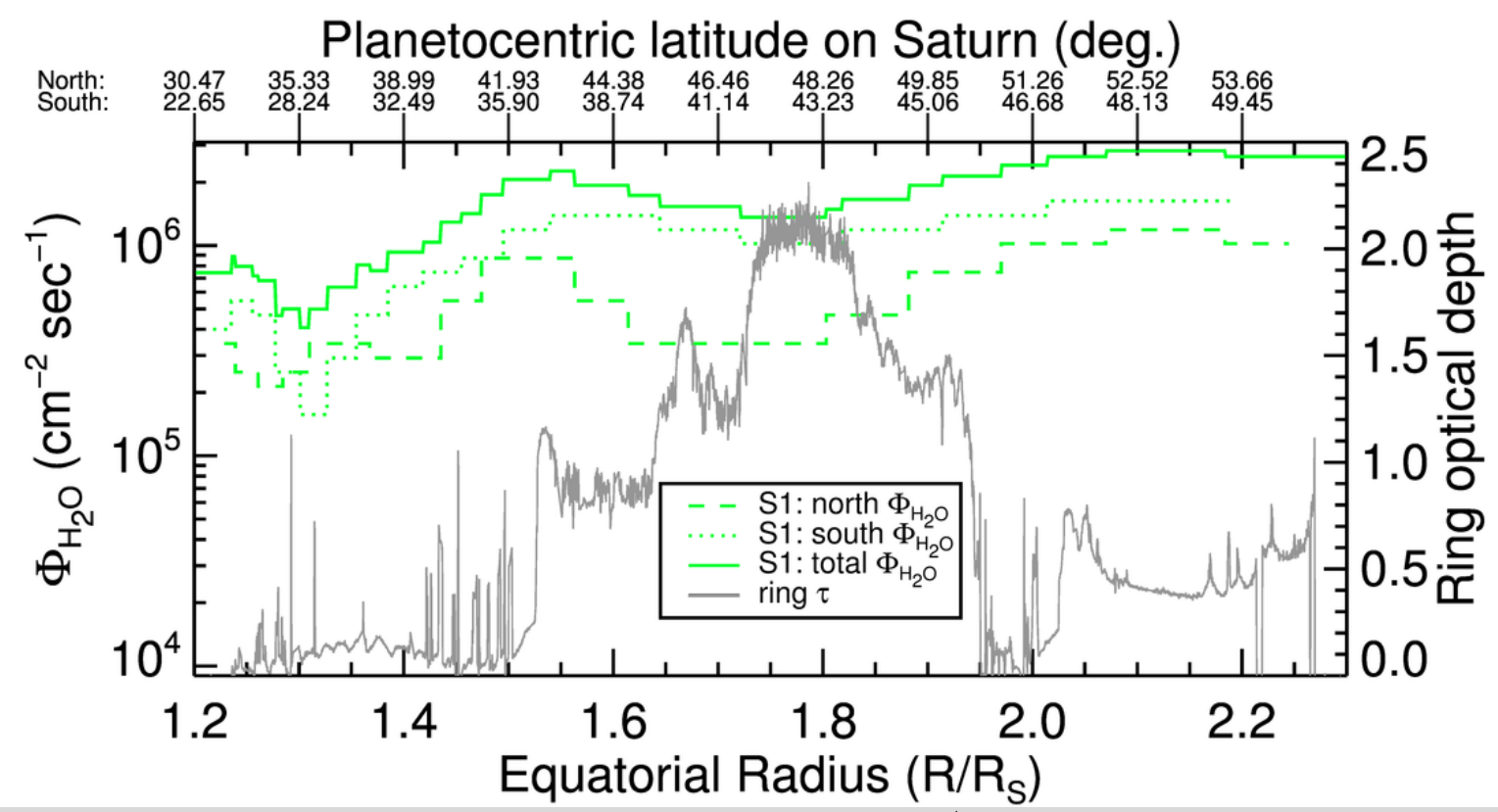

Figure 9. Maximum water influx estimates, based on $\mathrm{H}_{3}{ }^{+}$column densities derived from ring rain observations (Figure 3), and model comparisons. The influxes from Figure 8 are mapped along magnetic field lines to Saturn's ring plane (see text). Northern hemisphere water influxes (dashed green line) and southern hemisphere influxes (dotted green line) are shown as well as a combined hemispheric influx (solid green line). In addition, ring normal optical depths from IR ground based stellar occultation observations are plotted in gray.

Using Voyager measurements, Esposito et al. (1983) calculated a total ring mass of $2.8 \times 10^{19} \mathrm{~kg}$, roughly distributed as $6.25 \times 10^{18} \mathrm{~kg}$ in the A ring, $1.93 \times 10^{19} \mathrm{~kg}$ in the $\mathrm{B}$ ring, and $7.96 \times 10^{17} \mathrm{~kg}$ in the $\mathrm{C}$ ring. Later estimates include (4-7) $\times 10^{19} \mathrm{~kg}$ for the $\mathrm{B}$ ring (Robbins et al., 527 2010), as well as $4.9 \times 10^{18} \mathrm{~kg}$ and $(5-7) \times 10^{18} \mathrm{~kg}$ for the A ring (Spilker et al., 2004; Robbins et al., 2010). Converting integrated water influxes derived in Section 3.1. (Figure 9) to implied

529 mass loss rates gives $10 \mathrm{~kg} \mathrm{sec}^{-1}$ for the A ring, $8.8 \mathrm{~kg} \mathrm{sec}^{-1}$ for the $\mathrm{B}$ ring, and $2.7 \mathrm{~kg} \mathrm{sec}^{-1}$ for 530 the $\mathrm{C}$ ring. This calculation assumes a pure water influx in order to convert the Figure 9 values 531 to mass influx, and then integrates the derived mass influxes over the ring regions defined in 532 Table II of Esposito et al. (1983). If we use those mass loss rates and the Esposito et al. (1983)

533 ring mass estimates, and we make the (likely unrealistic) assumption that they hold constant in 534 time (with no other source replenishing the rings), then the maximum lifetimes of the A, B and C 
535 rings are $\sim 20, \sim 70$, and $\sim 9.3$ billion years, respectively. However, in addition to only 536 representing an upper limit, the impact of these lifetimes is further reduced by their reliance on a 537 number of key assumptions, which include a constant ring rain loss rate, and their neglect of the 538 possibility of narrow regions of enhanced ring loss (as such signatures would have been 539 smoothed out due to the latitude resolution of the ring rain observations). More thorough 540 estimates of ring lifetimes, using varying techniques, include 4.4-67 myr (Northrop and 541 Connerney, 1987), 100 myr (Cuzzi and Estrada, 1998; Salmon et al., 2010), and 4.5 billion years

542 (Canup, 2010). An overview of many past ring evolution studies is given by Chambers et al. 543 (2008).

\section{4. Discussion and Conclusion}

546 Quantifying the magnitude and temporal and spatial variability of the observed 547 exogenous source(s) of oxygen at Saturn is important for atmospheric chemistry and physics. 548 Dust particles can lead to localized heating of the upper atmosphere, thereby affecting 549 atmospheric dynamics (e.g., Rizk and Hunten, 1990), they can alter atmospheric photochemistry 550 through attenuation of solar UV radiation, and they can facilitate stratospheric haze formation by 551 providing condensation nuclei in the upper atmosphere (e.g., Moses et al., 2000, and references 552 therein). Similarly, vapor species can significantly alter stratospheric and ionospheric chemistry 553 (Connerney and Waite, 1984; Majeed and McConnell, 1991; Moses et al., 2000).

554 Indirect estimates of oxygen influx at Saturn use a measurement of one or more other 555 atmospheric parameters - typically electron density - in order to reproduce the observed 556 parameter(s). Previous indirect estimates include fluxes of $10^{7} \mathrm{OH} \mathrm{cm}^{-2} \mathrm{sec}^{-1}($ Shimizu, 1980), $557 \sim 10^{10} \mathrm{OH} \mathrm{cm} \sec ^{-1}$ (Chen, 1983), $\sim 4 \times 10^{7} \mathrm{H}_{2} \mathrm{O} \mathrm{cm} \mathrm{sec}^{-1}$ (Connerney and Waite, 1984), 2.2x10 
$\mathrm{H}_{2} \mathrm{O} \mathrm{cm} \mathrm{sec}^{-1}$ (Majeed and McConnell, 1991), (1-5) $\times 10^{7} \mathrm{~cm}^{-2} \mathrm{sec}^{-1}$ (Majeed and McConnell, 559 1996), and $5 \times 10^{6} \mathrm{H}_{2} \mathrm{O} \mathrm{cm}^{-2} \mathrm{sec}^{-1}$ (Moore et al., 2006). These estimates are all based on one or 560 more radio occultation measurements, or on Saturn Electrostatic Discharge (SED) observations, 561 and represent local influxes for the most part, as the observations are relatively sparse. Radio 562 occultations sample Saturn's dawn or dusk ionosphere at one point in time and therefore provide 563 little opportunity for constraining the possible temporal or spatial variation of the derived influx.

564 In contrast, SEDs use the low frequency cutoff from radio emissions generated by lightning in 565 Saturn's lower atmosphere to derive local time variations in peak ionospheric electron density.

566 SED measurements exist for both the Voyager (Kaiser et al., 1984) and Cassini (Fischer et al., 567 2011) eras, and indicate strong diurnal variations in electron density that models have not yet 568 been able to explain (Majeed and McConnell, 1996; Moore et al., 2012). The primary difficulty faced by previous model comparisons with SED-derived diurnal 570 variations in peak electron density is the extremely rapid buildup of ionization in the morning 571 hours implied by the measurements. For example, the net (i.e., production minus loss) electron 572 production rate between dawn and noon from SED measurements is between $\sim 9 \mathrm{~cm}^{-3} \mathrm{sec}^{-1}$ 573 (Cassini; Fischer et al., 2011) and $30-70 \mathrm{~cm}^{-3} \mathrm{sec}^{-1}$ (Voyager; Kaiser et al., 1984; Zarka, 1985), 574 whereas the peak overhead production rate due to solar EUV is $\sim 10 \mathrm{~cm}^{-3} \mathrm{sec}^{-1}$ (Moore et al., 575 2004). Therefore, an explanation of SED observations may require some sort of extreme 576 ionization enhancement process, such as due to a diurnal ionosphere-protonosphere exchange 577 (e.g. Connerney and Waite, 1984). One alternative explanation is that SEDs may be sampling 578 the sharp low-altitude ionospheric layers frequently seen in radio occultation electron density 579 profiles (Nagy et al., 2006; Kliore et al., 2009) rather than the canonical ionospheric peak. Such 580 layers are consistent with the presence of gravity waves in Saturn's lower thermosphere 
581 (Matcheva and Barrow, 2012), and can lead to narrow regions of electron density enhancements

582 without requiring any additional sources of ionization (Barrow and Matcheva, 2013). As the

583 atmospheric storms that give rise to SEDs tend to occur only at a limited set of specific latitudes

584 for currently unknown reasons (primarily $35^{\circ} \mathrm{S}$; Fischer et al., 2011), any ionospheric

585 explanation of SED-derived electron densities may also be local in nature.

586 Direct measurements of oxygen species in Saturn's upper atmosphere have proven

587 difficult. The first unambiguous direct detection of water in Saturn's upper atmosphere came

588 from the ISO (Feuchtgruber et al., 1997), which led to a derivation of global water influx of $589 \sim 1.5 \times 10^{6} \mathrm{H}_{2} \mathrm{O}$ molecules $\mathrm{cm}^{-2} \mathrm{sec}^{-1}$ (Moses et al., 2000), similar to later SWAS and Herschel

590 values (Bergin et al., 2000; Hartogh et al., 2011). Two ambiguous detections have been made in

591 the UV, one by the International Ultraviolet Explorer satellite (Winkelstein et al., 1983), and one

592 by HST, which indicated possible locally enhanced water abundance near $33^{\circ} \mathrm{S}$ latitude (Prangé

593 et al., 2006). Cassini's Composite Infrared Spectrometer (CIRS) instrument has also detected

594 weak $\mathrm{H}_{2} \mathrm{O}$ emission lines, which should allow a retrieval of latitudinal variation of water at

595 Saturn in the future (Bjoraker et al., 2010), with a preliminary analysis indicating a qualitatively

596 similar latitudinal trend to that derived by Moore et al. (2010).

597 Observations of $\mathrm{H}_{3}^{+}$allow for a significantly improved indirect estimate of external 598 oxygen influx at Saturn, as they can provide an extended latitude distribution of $\mathrm{H}_{3}{ }^{+}$column 599 densities in a single snapshot. Though uncertainty in some Saturn ionospheric photochemical 600 reactions remains, such as the effective rate of charge exchange between $\mathrm{H}^{+}$and vibrationally 601 excited $\mathrm{H}_{2}$, the water influxes derived here agree well with a number of different studies. They 602 represent a global influx that is comparable to the expected water vapor loss from Enceladus to 603 Saturn's atmosphere (Jurac and Richardson, 2007; Cassidy and Johnson, 2010; Hartogh et al., 
604 2011; Fleshman et al., 2012), and a ring derived influx with a magnitude and hemispheric 605 asymmetry consistent with earlier predictions (e.g., Connerney, 1986) and ring atmosphere 606 calculations (Luhmann et al., 2006; Tseng et al., 2010). Local peak influxes are at latitudes that 607 map magnetically to instability radii in Saturn's rings, long predicted to be a prime location for 608 siphoning material from Saturn's rings into its atmosphere (e.g., Northrop and Hill, 1982, 1983; 609 Connerney, 1986). The latitudinally averaged influx is in good agreement with that derived from 610 ISO measurements (Feuchtgruber et al., 1997), while the latitudinal variations in influx are 611 likely not drastic enough to conflict with the constraints derived by Moses et al. (2000).

612 Taken together, the above agreements with previous work demonstrate an internal 613 consistency which makes the derived values more convincing. However, there are also a number 614 of limitations to the current approach. These include: a lack of self-consistent $\mathrm{H}_{3}{ }^{+}$temperatures 615 from the ring rain observations, an insufficient latitude resolution for direct comparisons with 616 predicted narrow regions of enhanced ring-derived influx (e.g., Connerney and Waite, 1984; 617 Connerney, 1986), and an assumption of constant influx. For example, electron column contents 618 from radio occultation observations are used to discount solutions with large water influxes (e.g., $619>10^{7} \mathrm{~cm}^{-2} \mathrm{sec}^{-1}$; Figure 5). However, if the external water influx at Saturn is time-variable (e.g., 620 Moore and Mendillo, 2007), as opposed to constant as considered here, then this argument would 621 be weakened, as the radio occultations of electron densities and the $\mathrm{H}_{3}{ }^{+}$observations were taken 622 years apart and sample different local times. Furthermore, radio occultations sample a range of 623 latitudes while relying on the assumption of a horizontally stratified ionosphere, and 624 consequently would not be expected to detect narrowly confined ionospheric perturbations, such 625 as from a region of enhanced water influx. Therefore, despite the discrepancy between model 626 results and measured column electron densities for large water influxes shown in Figure 5, the 
627 "high" water influx family of solutions shown in Figure 4 cannot be dismissed unequivocally

628 without further observational evidence.

\subsection{Radio Occultation Observations}

There is a discrepancy between the latitudinal variation of water influxes calculated here

632 and that derived based on comparisons with Cassini radio occultation measurements (Figure 8;

633 also Moore et al., 2010). In fact, the ring rain water influxes from this work lead to a mid-

634 latitude trend in electron density - a decrease with increasing latitude - that is counter to what

635 has been observed (Kliore et al., 2009). There are a number of possible explanations for this

636 fact. First, while the observed neutral temperatures seem to increase with latitude, the STIM-

637 derived temperature differentials also increase with latitude (Figure 2). This leads to a prediction

638 for $\mathrm{H}_{3}{ }^{+}$temperatures that is flat or decreasing with latitude, and an inverse trend in $\mathrm{H}_{3}{ }^{+}$column

639 densities. Consequently, the resulting water influx estimates also increase with latitude. Future

640 ring rain observations should be able to address this possibility, as the O'Donoghue et al. (2013)

641 results were based on only $\sim 2$ hours of data; longer integrations will allow for self-consistent $\mathrm{H}_{3}{ }^{+}$

642 temperature and density measurements. Second, there are only 5 published Cassini radio

643 occultations within the ring rain latitudes, whereas 31 radio occultations were used to derive the

644 latitudinal trend in electron density. This means that the overall trend was anchored by the 18

645 equatorial radio occultation profiles and increased with latitude to match the 8 high-latitude

646 profiles. Further mid-latitude Cassini radio occultations will help to establish the strength of the

647 latitudinal electron density trend there, and may reveal localized minima and maxima with the

648 increased latitude resolution, if present. Finally, the Cassini radio occultations occurred 3-6

649 years prior to the ring rain observations (and thus to a different season and solar cycle phase), 
650 and so it may not be reasonable to expect that one set of parameters should reproduce both data

651 sets simultaneously.

\section{4.2. Summary}

654 Based on model comparisons with observations of mid- and low-latitude $\mathrm{H}_{3}{ }^{+}$emission at 655 Saturn, detected recently for the first time, we have estimated maximum ring-derived water 656 influxes as a function of latitude on the planet and as a function of radius in the ring plane. We 657 find globally averaged maximum ring-derived water influxes of (1.6-12) $\times 10^{5} \mathrm{~cm}^{-2} \mathrm{sec}^{-1}$, which 658 corresponds to a maximum total global rate of water molecules from Saturn's rings to its 659 atmosphere of $(1.0-6.8) \times 10^{26} \mathrm{sec}^{-1}$. Though they represent a non-unique solution, our 660 distribution of influxes is in good agreement with a range of predictions resulting from different 661 aspects of Saturn system science. Future observations of mid-latitude $\mathrm{H}_{3}{ }^{+}$at Saturn would allow 662 for significant improvements to this work, including: (1) an increased latitude resolution (as 663 Saturn tilts more towards Earth in its approach to northern summer solstice); (2) an examination 664 of any seasonal or temporal differences; and (3) a self-consistent measurement of $\mathrm{H}_{3}{ }^{+}$ 665 temperature. This last point is extremely important, because the necessary fundamental 666 assumption in this study is that the observed structure in $\mathrm{H}_{3}{ }^{+}$emission is caused by variations in $667 \mathrm{H}_{3}{ }^{+}$column density (driven by a water influx), and not by variations in $\mathrm{H}_{3}{ }^{+}$temperatures. 668 Determination of the external oxygen influx at Saturn is relevant for a wide range of atmospheric 669 chemistry and dynamics. As the timescale for diffusion through the upper atmosphere is rapid 670 compared to the lower atmosphere, observing the upper atmospheric signatures of such an influx 671 is key for gauging its spatial and temporal variability. 


\section{Acknowledgements}

674 We acknowledge the contribution of the International Space Sciences Institute (ISSI) in Bern,

675 Switzerland, for hosting and funding the ISSI International Team on "Comparative Jovian

676 Aeronomy", and the constructive discussions by colleagues attending the meetings. Funding for

677 this work at Boston University comes from NASA Grants NNX13AG21G and NNX13AG57G.

678 J.O'D. was supported by the UK Science and Technology Facilities Council (STFC) through a

679 PhD Studentship. I.M.W. and M.G. were partially funded by STFC through the Consolidated

Grant to Imperial College London.

\section{References}

Barrow, D. J., and K. I. Matcheva (2013), Modeling the effect of atmospheric gravity waves on Saturn's ionosphere, Icarus, 224(1), 32-42, doi:10.1016/j.icarus.2013.01.027.

Bergin, E. A. et al. (2000), Submillimeter Wave Astronomy Satellite Observations of Jupiter and Saturn: Detection of $557 \mathrm{GHz}$ Water Emission from the Upper Atmosphere, Astrophys. J., 4(539), 147-150.

Bjoraker, G., R. K. Achterberg, and D. E. Jennings (2010), Cassini / CIRS observations of water vapor in Saturn 's stratosphere, in EPSC Abstracts, vol. 5, p. 9007.

Broadfoot, A. L. et al. (1981), Extreme Ultraviolet Observations from Voyager 1 Encounter with Saturn, Science (80-. )., 212(4491), 206-211.

Bunce, E. J., C. S. Arridge, S. W. H. Cowley, and M. K. Dougherty (2008), Magnetic field structure of Saturn's dayside magnetosphere and its mapping to the ionosphere: Results from ring current modeling, J. Geophys. Res., 113(A2), A02207, doi:10.1029/2007JA012538.

Burton, M. E., M. K. Dougherty, and C. T. Russell (2010), Saturn's internal planetary magnetic field, Geophys. Res. Lett., 37(24), n/a-n/a, doi:10.1029/2010GL045148.

Canup, R. M. (2010), Origin of Saturn's rings and inner moons by mass removal from a lost Titan-sized satellite, Nature, 468(7326), 943-6, doi:10.1038/nature09661.

Capone, L. A., R. C. Whitten, S. S. Prasad, and J. Dubach (1977), The Ionospheres of Saturn, Uranus, and Neptune, Astrophys. J., 215, 977-983.

Cassidy, T. A., and R. E. Johnson (2010), Collisional spreading of Enceladus' neutral cloud, Icarus, 209(2), 696703, doi:10.1016/j.icarus.2010.04.010.

Chambers, L. S., J. N. Cuzzi, E. Asphaug, J. Colwell, and S. Sugita (2008), Hydrodynamical and radiative transfer modeling of meteoroid impacts into Saturn's rings, Icarus, 194(2), 623-635, doi:10.1016/j.icarus.2007.11.017.

Chen, R. H. (1983), Saturn's ionosphere: A corona of ice particles?, Moon Planets, 28, 37-41.

Connerney, J. (2013), Saturn's ring rain, Nature, 496(7444), 178-179.

Connerney, J., and J. Waite (1984), New model of Saturn's ionosphere with an influx of water from the rings, Nature, 312, 136-138.

Connerney, J. E. P. (1986), Magnetic Connection for Saturn's Rings and Atmosphere, Geophys. Res. Lett., 13(8), 773-776.

Cravens, T. E. (1987), Vibrationally Excited Molecular Hydrogen in the Upper Atmosphere of Jupiter, J. Geophys. Res., 92(5), 11,083-11,100.

Cuzzi, J. N., and P. R. Estrada (1998), Compositional Evolution of Saturn's Rings Due to Meteoroid Bombardment, , 35, 1-35. 
Cuzzi, J. N. et al. (2010), An evolving view of Saturn's dynamic rings., Science, 327(5972), 1470-5, doi:10.1126/science.1179118.

Esposito, L. W., M. O'Callaghan, and R. a. West (1983), The structure of Saturn's rings: Implications from the Voyager stellar occultation, Icarus, 56(3), 439-452, doi:10.1016/0019-1035(83)90165-3.

Festou, M. C., and S. K. Atreya (1982), Voyager ultraviolet stellar occultation measurements of the composition and thermal profiles of the Saturnian upper atmosphere, Geophys. Res. Lett., 9(10), 1147-1150.

Feuchtgruber, H., E. Lellouch, and T. De Graauw (1997), External supply of oxygen to the atmospheres of the giant planets, Nature, 389(September), 159-162.

Fischer, G., D. a. Gurnett, P. Zarka, L. Moore, and U. a. Dyudina (2011), Peak electron densities in Saturn's ionosphere derived from the low-frequency cutoff of Saturn lightning, J. Geophys. Res., 116(A4), A04315, doi:10.1029/2010JA016187.

Fleshman, B. L., P. A. Delamere, F. Bagenal, and T. Cassidy (2012), The roles of charge exchange and dissociation in spreading Saturn's neutral clouds, J. Geophys. Res., 117(E5), E05007, doi:10.1029/2011JE003996.

French, R., and P. D. Nicholson (2000), Saturn's Rings II Particle Sizes Inferred from Stellar Occultation Data, Icarus, 145(2), 502-523, doi:10.1006/icar.2000.6357.

Gérard, J.-C., B. Bonfond, J. Gustin, D. Grodent, J. T. Clarke, D. Bisikalo, and V. Shematovich (2009), Altitude of Saturn's aurora and its implications for the characteristic energy of precipitated electrons, Geophys. Res. Lett., 36(2), n/a-n/a, doi:10.1029/2008GL036554.

Hartogh, P. et al. (2011), Direct detection of the Enceladus water torus with Herschel, Astron. Astrophys., 532, L2, doi:10.1051/0004-6361/201117377.

Hubbard, W. B. et al. (1997), Structure of Saturn's Mesosphere from the 28 Sgr Occultations, Icarus, 130, 404-425.

Huestis, D. L. (2008), Hydrogen collisions in planetary atmospheres, ionospheres, and magnetospheres, Planet. Space Sci., 56(13), 1733-1743, doi:10.1016/j.pss.2008.07.012.

Huestis, D. L., S. W. Bougher, J. L. Fox, M. Galand, R. E. Johnson, J. I. Moses, and J. C. Pickering (2008), Cross Sections and Reaction Rates for Comparative Planetary Aeronomy, Space Sci. Rev., 139(1-4), 63-105, doi:10.1007/s11214-008-9383-7.

Johnson, R. E. et al. (2006), Production, ionization and redistribution of O2 in Saturn's ring atmosphere, Icarus, 180(2), 393-402, doi:10.1016/j.icarus.2005.08.021.

Jurac, S., and J. D. Richardson (2007), Neutral cloud interaction with Saturn's main rings, Geophys. Res. Lett., 34(8), L08102, doi:10.1029/2007GL029567.

Kaiser, M. L., M. D. Desch, and J. E. P. Connerney (1984), Saturn's ionosphere: Inferred electron densities, J. Geophys. Res., 89(A4), 2371, doi:10.1029/JA089iA04p02371.

Kliore, A. J., I. R. Patel, G. F. Lindal, D. N. Sweetnam, and H. B. Hotz (1980), Structure of the Ionosphere and Atmosphere of Saturn From Pioneer 11 Saturn Radio Occultation, J. Geophys. Res., 85(A11), 5857-5870.

Kliore, A. J., A. F. Nagy, E. A. Marouf, A. Anabtawi, E. Barbinis, D. U. Fleischman, and D. S. Kahan (2009), Midlatitude and high-latitude electron density profiles in the ionosphere of Saturn obtained by Cassini radio occultation observations, J. Geophys. Res., 114(A4), A04315, doi:10.1029/2008JA013900.

Koskinen, T. T., B. R. Sandel, R. V. Yelle, F. J. Capalbo, G. M. Holsclaw, W. E. McClintock, and S. Edgington (2013), The density and temperature structure near the exobase of Saturn from Cassini UVIS solar occultations, Icarus, 226(2), 1318-1330, doi:10.1016/j.icarus.2013.07.037.

Lindal, G. F., D. N. Sweetnam, and V. R. Eshleman (1985), The Atmosphere of Saturn: An Analysis of the Voyager Radio Occultation Measurements, Astron. J., 90(6), 1136-1146.

Luhmann, J. G., R. E. Johnson, R. L. Tokar, S. a. Ledvina, and T. E. Cravens (2006), A model of the ionosphere of Saturn's rings and its implications, Icarus, 181(2), 465-474, doi:10.1016/j.icarus.2005.11.022.

Majeed, T., and J. McConnell (1991), The upper ionospheres of Jupiter and Saturn, Planet. Space Sci., 39(12), $1715-1732$.

Majeed, T., and J. McConnell (1996), Voyager electron density measurements on Saturn: Analysis with a time dependent ionospheric model, J. Geophys. Res., 101, 7589-7598.

Majeed, T., J. McConnell, and R. Yelle (1990), Vibrationally excited H2 in the upper atmosphere of Saturn, Adv. Sp. Res., 10(1), 131-134.

Majeed, T., J. McConnell, and R. Yelle (1991), Vibrationally excited H2 in the outer planets thermosphere: Fluorescence in the Lyman and Werner bands, Planet. Space Sci., 39(11), 1591-1606.

Matcheva, K. I., and D. J. Barrow (2012), Small-scale variability in Saturn's lower ionosphere, Icarus, 221(2), 525543, doi:10.1016/j.icarus.2012.08.022.

McElroy, M. (1973), The ionospheres of the major planets, Space Sci. Rev., 14, 460-473. 
Melin, H., S. Miller, T. Stallard, L. M. Trafton, and T. R. Geballe (2007), Variability in the H3+ emission of Saturn: Consequences for ionisation rates and temperature, Icarus, 186(1), 234-241, doi:10.1016/j.icarus.2006.08.014.

Melin, H., T. Stallard, S. Miller, J. Gustin, M. Galand, S. V. Badman, W. R. Pryor, J. O’Donoghue, R. H. Brown, and K. H. Baines (2011), Simultaneous Cassini VIMS and UVIS observations of Saturn's southern aurora: Comparing emissions from H, $\mathrm{H} 2$ and $\mathrm{H} 3+$ at a high spatial resolution, Geophys. Res. Lett., 38(15), n/a-n/a, doi:10.1029/2011GL048457.

Miller, S., R. D. Joseph, and J. Tennyson (1990), Infrared Emissions of H3+ in the Atmosphere of Jupiter in the 2.1 and 4.0 micron Region, Astrophys. J., 360, L55-L58.

Miller, S. et al. (2000), The role of H3+ in planetary atmospheres, Philos. Trans. R. Soc. A Math. Phys. Eng. Sci., 358, 2485-2502.

Miller, S., T. Stallard, H. Melin, and J. Tennyson (2010), H3+ cooling in planetary atmospheres, Faraday Discuss., 147, 283, doi:10.1039/c004152c.

Moore, L., and M. Mendillo (2007), Are plasma depletions in Saturn's ionosphere a signature of time-dependent water input?, Geophys. Res. Lett., 34(12), L12202, doi:10.1029/2007GL029381.

Moore, L., A. F. Nagy, A. J. Kliore, I. Müller-Wodarg, J. D. Richardson, and M. Mendillo (2006), Cassini radio occultations of Saturn's ionosphere: Model comparisons using a constant water flux, Geophys. Res. Lett., 33(22), L22202, doi:10.1029/2006GL027375.

Moore, L., M. Galand, I. Mueller-Wodarg, R. Yelle, and M. Mendillo (2008), Plasma temperatures in Saturn's ionosphere, J. Geophys. Res., 113(A10), A10306, doi:10.1029/2008JA013373.

Moore, L., M. Galand, I. Mueller-Wodarg, and M. Mendillo (2009), Response of Saturn's ionosphere to solar radiation: Testing parameterizations for thermal electron heating and secondary ionization processes, Planet. Space Sci., 57(14-15), 1699-1705, doi:10.1016/j.pss.2009.05.001.

Moore, L., I. Mueller-Wodarg, M. Galand, A. Kliore, and M. Mendillo (2010), Latitudinal variations in Saturn's ionosphere: Cassini measurements and model comparisons, J. Geophys. Res., 115(A11), A11317, doi:10.1029/2010JA015692.

Moore, L., G. Fischer, I. Müller-Wodarg, M. Galand, and M. Mendillo (2012), Diurnal variation of electron density in Saturn's ionosphere: Model comparisons with Saturn Electrostatic Discharge (SED) observations, Icarus, 221(2), 508-516, doi:10.1016/j.icarus.2012.08.010.

Moore, L. E., M. Mendillo, I. C. F. Müller-Wodarg, and D. L. Murr (2004), Modeling of global variations and ring shadowing in Saturn's ionosphere, Icarus, 172(2), 503-520, doi:10.1016/j.icarus.2004.07.007.

Moses, J., and S. Bass (2000), The effects of external material on the chemistry and structure of Saturn's ionosphere, J. Geophys. Res., 105(1999), 7013-7052.

Moses, J., B. Bézard, E. Lellouch, G. R. Gladstone, H. Feuchtgruber, and M. Allen (2000), Photochemistry of Saturn's Atmosphere II. Effects of an Influx of External Oxygen, Icarus, 145(1), 166-202, doi:10.1006/icar.1999.6320.

Müller-Wodarg, I. C. F., L. Moore, M. Galand, S. Miller, and M. Mendillo (2012), Magnetosphere-atmosphere coupling at Saturn: 1 - Response of thermosphere and ionosphere to steady state polar forcing, Icarus, 221(2), 481-494, doi:10.1016/j.icarus.2012.08.034.

Nagy, A. F. et al. (2006), First results from the ionospheric radio occultations of Saturn by the Cassini spacecraft, $J$. Geophys. Res., 111(A6), A06310, doi:10.1029/2005JA011519.

Neale, L., S. Miller, and J. Tennyson (1996), Spectroscopic Properties of the H3+ Molecule: A New Calculated Line List, Astrophys. J., 464, 516-520.

Nicholson, P., R. French, E. Tollestrup, J. Cuzzi, J. Harrington, K. Matthews, O. Perkovic, and R. Stover (2000), Saturn's Rings I Optical Depth Profiles from the 28 Sgr Occultation, Icarus, 145(2), 474-501, doi:10.1006/icar.2000.6356.

Northrop, G., and J. R. Hill (1982), Stability of Negatively Charged Dust Graings in Saturn's Ring Plane, J. Geophys. Res., 87(A8), 6045-6051.

Northrop, T. G., and J. E. P. Connerney (1987), A Micrometeorite Erosion Model and the Age of Saturn's Rings, Icarus, 70, 124-137.

Northrop, T. G., and J. R. Hill (1983), The Inner Edge of Saturn's B Ring, J. Geophys. Res., 88(A8), $6102-6108$.

O’Donoghue, J., T. S. Stallard, H. Melin, G. H. Jones, S. W. H. Cowley, S. Miller, K. H. Baines, and J. S. D. Blake (2013), The domination of Saturn's low-latitude ionosphere by ring “rain," Nature, 496(7444), 193-195, doi:10.1038/nature12049. 
O’Donoghue, J., T. S. Stallard, H. Melin, S. W. H. Cowley, S. V. Badman, L. Moore, S. Miller, C. Tao, K. H. Baines, and J. S. D. Blake (2014), Conjugate observations of Saturn's northern and southern aurorae, Icarus, 229, 214-220, doi:10.1016/j.icarus.2013.11.009.

Prangé, R., T. Fouchet, R. Courtin, J. E. P. Connerney, and J. C. McConnell (2006), Latitudinal variation of Saturn photochemistry deduced from spatially-resolved ultraviolet spectra, Icarus, 180(2), 379-392, doi:10.1016/j.icarus.2005.11.005.

Rizk, B., and D. M. Hunten (1990), Solar heating of the Uranian mesopause by dust of ring origin, Icarus, 88(2), 429-447, doi:10.1016/0019-1035(90)90093-O.

Robbins, S. J., G. R. Stewart, M. C. Lewis, J. E. Colwell, and M. Sremčević (2010), Estimating the masses of Saturn's A and B rings from high-optical depth N-body simulations and stellar occultations, Icarus, 206(2), 431-445, doi:10.1016/j.icarus.2009.09.012.

Salmon, J., S. Charnoz, a. Crida, and a. Brahic (2010), Long-term and large-scale viscous evolution of dense planetary rings, Icarus, 209(2), 771-785, doi:10.1016/j.icarus.2010.05.030.

Sandel, A. B. R. et al. (1982), Extreme Ultraviolet Observations from the Voyager 2 Encounter with Saturn, Science (80-. )., 215(4532), 548-553.

Seidelmann, P. K. et al. (2007), Report of the IAU/IAG Working Group on cartographic coordinates and rotational elements: 2006, Celest. Mech. Dyn. Astron., 98(3), 155-180, doi:10.1007/s10569-007-9072-y.

Shemansky, D. E., and X. Liu (2012), Saturn upper atmospheric structure from Cassini EUV and FUV occultations, Can. J. Phys., 90(8), 817-831, doi:10.1139/p2012-036.

Shimizu, M. (1980), Strong interaction between the ring system and the ionosphere of Saturn, Moon Planets, 22, 521-522.

Smith, G. R., D. E. Shemansky, J. B. Holberg, A. L. Broadfoot, and B. R. Sandel (1983), Saturn's upper atmosphere from the Voyager 2 EUV solar and stellar occultations, J. Geophys. Res., 88(A11), 8667-8678.

Spilker, L. J., S. Pilorz, A. L. Lane, R. M. Nelson, B. Pollard, and C. T. Russell (2004), Saturn A ring surface mass densities from spiral density wave dispersion behavior, Icarus, 171(2), 372-390, doi:10.1016/j.icarus.2004.05.016.

Stallard, T., S. Miller, G. Millward, and R. D. Joseph (2002), On the Dynamics of the Jovian Ionosphere and Thermosphere II. The Measurement of H3+ Vibrational Temperature, Column Density, and Total Emission, Icarus, 156(2), 498-514, doi:10.1006/icar.2001.6793.

Stallard, T. S., H. Melin, S. Miller, S. V. Badman, R. H. Brown, and K. H. Baines (2012), Peak emission altitude of Saturn's H3+ aurora, Geophys. Res. Lett., 39(15), 1-5, doi:10.1029/2012GL052806.

Tokar, R. L. et al. (2005), Cassini observations of the thermal plasma in the vicinity of Saturn's main rings and the F and G rings, Geophys. Res. Lett., 32(14), 1-5, doi:10.1029/2005GL022690.

Tseng, W.-L., W.-H. Ip, R. E. Johnson, T. A. Cassidy, and M. K. Elrod (2010), The structure and time variability of the ring atmosphere and ionosphere, Icarus, 206(2), 382-389, doi:10.1016/j.icarus.2009.05.019.

Vervack, R. J., and J. I. Moses (2013), Saturn's upper atmosphere during the Voyager era: Reanalysis and modeling of the UVS occultations, Icarus, submitted.

Waite, J. H., T. E. Cravens, W. Ip, W. T. Kasprzak, J. G. Luhmann, R. L. Mcnutt, H. B. Niemann, R. V Yelle, S. A. Ledvina, and S. Scherer (2005), Oxygen Ions Observed Near Saturn's A Ring, Science (80-. )., 307(February), $1260-1262$.

Winkelstein, P., J. Caldwell, S. J. Kim, G. E. Hunt, and V. Moore (1983), A Determination of the Composition of the Saturnian Stratosphere Using the IUE, Icarus, 54, 309-318.

Woods, T. N. (2008), Recent advances in observations and modeling of the solar ultraviolet and X-ray spectral irradiance, Adv. Sp. Res., 42(5), 895-902, doi:10.1016/j.asr.2007.09.026.

Woods, T. N. et al. (2000), TIMED Solar EUV Experiment, Phys. Chem. Earth C, 25(5-6), 393-396.

Woods, T. N., F. G. Eparvier, S. M. Bailey, P. C. Chamberlin, J. Lean, G. J. Rottman, S. C. Solomon, W. K. Tobiska, and D. L. Woodraska (2005), Solar EUV Experiment (SEE): Mission overview and first results, $J$. Geophys. Res., 110(A1), A01312, doi:10.1029/2004JA010765.

Zarka, P. (1985), Directivity of Saturn Electrostatic Discharges and Ionospheric Implications, Icarus, 61, 508-520.

Zebker, H. A., E. A. Marouf, and G. L. Tyler (1985), Saturn's Rings: Particle Size Distributions for Thin Layer Models, Icarus, 64, 531-548. 